\title{
REVISIÓN TAXONÓMICA Y DISTRIBUCIÓN GEOGRÁFICA DE EPHEDRA (EPHEDRACEAE) EN MÉXICO
}

\author{
Lorena Villanueva-Almanza ${ }^{1}$ y Rosa María Fonseca \\ Universidad Nacional Autónoma de México, Facultad de Ciencias, Laboratorio de \\ Plantas Vasculares, Apdo. postal 70.282, 04510, México, D.F. \\ 1Autor para la correspondencia: lorevial@ciencias.unam.mx
}

\section{RESUMEN}

El objetivo de este trabajo es dar conocer los representantes del género Ephedra en México y su distribución a nivel nacional. En el territorio de la República existen ocho especies de Ephedra en el norte y centro del país: Ephedra antisyphilitica, E. aspera, E. californica, E. compacta, E. nevadensis, E. pedunculata, E. torreyana y E. trifurca. Ephedra compacta es la única endémica de México y la de mayor distribución en su superficie. Se elaboró una clave, descripciones y mapas de repartición geográfica de las especies. Se encontró que las poblaciones de Ephedra en México se presentan entre los 0 y $2800 \mathrm{~m}$ de altitud, en matorral xerófilo, pastizal y bosque espinoso, principalmente.

Palabras clave: distribución geográfica, Ephedra, México, taxonomía.

\begin{abstract}
This research seeks to determine the number of species of Ephedra in Mexico and their distributions in the national territory. Eight species of Ephedra are distributed in North and Central Mexico: Ephedra antisyphilitica, E. aspera, E. californica, E. compacta, E. nevadensis, E. pedunculata, E. torreyana and E. trifurca. Ephedra compacta is the only species endemic to Mexico and the one with the most widely distribution over the country. A key, descriptions for each of the species, and distribution maps are presented. Ephedra species are found between 0 and $2800 \mathrm{~m}$ above sea level, mostly in xerophytic scrublands, grasslands, and thorn forests.
\end{abstract}

Key words: Ephedra, geographic distribution, Mexico, taxonomy. 


\section{INTRODUCCIÓN}

El género Ephedra L. (Ephedraceae) consta de aproximadamente 60 especies distribuidas en las regiones áridas y semiáridas del mundo.

No existe evidencia precisa acerca de la ubicación cronológica del origen de Ephedra, aunque se sostienen dos hipótesis: una la sitúa en el Oligoceno-Mioceno de acuerdo con un análisis de fechamiento molecular; mientras que la otra supone su génesis en el Cretácico (Rydin et al., 2006). Los cálculos de fechamiento a través de un reloj molecular, indican que el género pudo haberse originado hace 8 a 32 millones de años (Yang et al., 2005). Los estudios filogenéticos más recientes indican una división geográfica basal que engendra al clado americano a partir del linaje primitivo del Viejo Mundo. Dentro del primero se reconocen dos subclados, el norteamericano y el sudamericano (Hollander et al., 2009).

Se conocen escasos macrofósiles relacionados con Ephedra, los cuales han sido ubicados en los géneros Ephedrites Saporta, Chaoyangia Duan y Eoanthus Krassilov (Yang et al., 2005), aunque el registro palinológico es relativamente abundante. Recientemente se encontraron macrofósiles que datan del Cretácico Inferior, en la formación de Yixian, en China, a partir de los cuales en 2005 se describió Ephedra archaerhytidosperma Y. Yang, B. Y. Geng, D. L. Dilcher, Z. D. Chen y T. A. Lott. (Yang et al., 2005), la cual presenta características muy similares a las de la actual E. rhytidosperma Pachomova, endémica de China.

El género Ephedra fue publicado por Linneo en 1753, la especie tipo es Ephedra distachya L. La monografía inicial del grupo, donde se describe por primera vez a un representante norteamericano, Ephedra antisyphilitica, es la de Meyer (1846). Watson publicó E. trifurca en 1871, ocho años después dio a conocer otras tres especies, E. californica, E. nevadensis y E. torreyana y más tarde, a partir de las notas de Engelmann, a E. aspera y E. pedunculata (Cutler, 1939). Posteriormente Stapf (1889) revisó el género incluyendo 31 especies distribuidas en tres secciones con base en los caracteres de las brácteas: Alatae con brácteas membranáceas, secas y aladas, Asarca con brácteas secas, no aladas y Pseudobaccateae (=Ephedra) con brácteas carnosas, no aladas. De acuerdo con Ickert-Bond y Wojciechowski (2004), las tres secciones propuestas por Stapf (1889) no forman grupos monofiléticos; sin embargo, los caracteres que tomó en cuenta son útiles para determinar especies.

Cutler (1939) publicó una revisión de Ephedra en Norteamérica, en la cual por primera vez se incluye a E. compacta, descrita en 1909. Stevenson (1993) retomó el género en la Flora de Norteamérica reconociendo 12 especies para esa región. Otros trabajos que han aportado conocimiento sobre el grupo son el de Martens (1971) 
acerca de la anatomía, citología y embriología de las Gnetales y el de Pant y Verma (1974), donde se hace un análisis de las características de la epidermis y la cutícula en varias especies.

Para México, Standley (1923) en "Trees and shrubs of Mexico”, consideró seis especies: E. antisyphilitica, E. aspera, E. californica, E. compacta, E. pedunculata y E. trifurca; Johnston, en 1943, en su trabajo Plants of Coahuila, Eastern Chihuahua, and adjoining Zacatecas and Durango, proporcionó una breve descripción de E. antisyphilitica, E. compacta, E. pedunculata, E. torreyana y E. trifurca. Valdés y Flores (1987) en "Las gimnospermas en la flora halófila y gipsófila de México”, incluyen una lista de seis especies de Ephedra. Conzatti (1988) en la "Flora taxonómica mexicana”, reconoció a los mismos componentes registrados por Standley (1923), con una clave de determinación y una lista breve de características de cada una de estas especies. Wendt (1993) publicó la variedad Ephedra torreyana var. powelliorum del oriente del estado de Chihuahua. Medina y Dávila (1997), en la Flora del Valle de Tehuacán-Cuicatlán, consideraron a Ephedra compacta. Méndez (1998) estudió la distribución ecológica de E. antisyphilitica, E. aspera, E. compacta, E. pedunculata y E. trifurca en el Altiplano de San Luis Potosí.

Desde la antigüedad, especialmente en el contintente asiático, varios representantes de Ephedra han sido utilizados con fines medicinales contra la tos, como antisifilíticos, antipiréticos, estimulantes de la circulación y antihistamínicos. El principio activo conocido como efedrina está presente únicamente en las especies de Asia; las de América producen sólo pseudoefedrina a niveles apenas detectables (Caveney et al., 2001).

Desde el punto de vista ecológico, según Hollander y colaboradores (2009), las semillas de especies con brácteas carnosas y suculentas, como Ephedra antisyphilitica, son dispersadas por aves; las que sobresalen entre pequeñas brácteas secas, por ejemplo E. aspera y E. californica lo son por roedores; mientras que aquellas con brácteas secas y semillas largas como E. torreyana y E. trifurca se esparcen por medio del viento.

En este trabajo se presenta información sobre la diversidad y la distribución geográfica de Ephedra en México.

\section{MÉTODOS}

Se hizo una investigación bibliográfica acerca de Ephedra en México; se realizó trabajo de campo en los estados de San Luis Potosí y Puebla, donde se 
observaron especies de Ephedra en su ambiente y se recolectaron ejemplares para depositarlos en los herbarios. Se revisó la determinación y se anotó la información correspondiente a los ejemplares en los siguientes herbarios: Herbario Nacional en el Instituto de Biología (MEXU), el de la Escuela Nacional de Ciencias Biológicas del Instituto Politécnico Nacional (ENCB), el de la Universidad Autónoma Metropolitana, unidad Iztapalapa (UAMIZ) y el del Centro de Investigaciones de Zonas Desérticas de San Luis Potosí (SLPM). Las observaciones realizadas en estas colecciones permitieron corroborar o corregir la identificación de los especímenes, ampliar las descripciones ya existentes de las especies y generar una base de datos con localidad de colecta, latitud, longitud, altitud, tipo de vegetación, vegetación asociada, tamaño y sexo de la planta, así como colector, su número y fecha. Con la información del lugar obtenida de los ejemplares de herbario se calcularon las coordenadas de los sitios correspondientes. Posteriormente, con el uso de un sistema de información geográfica (Arc Gis) se obtuvieron mapas digitalizados de la distribución geográfica registrada en el territorio de la República de cada una de las especies mexicanas.

\section{RESULTADOS}

Se registró la presencia de ocho especies del género Ephedra en México: $E$. antisyphilitica, E. aspera, E. californica, E. compacta, E. nevadensis, E. pedunculata, E. torreyana y E. trifurca; en la base de datos se generaron 486 registros y se representó en mapas la información relativa a su distribución geográfica. Algunos ejemplares estériles no fueron incluidos en este trabajo, ya que no pudieron ser determinados con seguridad; mientras que otros, aún carentes de órganos de reproducción, se adjudicaron a alguna especie cuando sus características aseguraban una identificación correcta.

Ephedra L., Sp. Pl. 2: 1040. 1753. (Gen. Pl. ed. 5, 462. 1754).

Arbustos erectos o compactos o trepadores o extendidos y postrados, dioicos o rara vez monoicos, con eustele, con vasos y traqueidas. Raíces generalmente fibrosas o con un eje central notoriamente más ancho y raíces laterales. Tallos muy ramificados, fotosintéticos, cilíndricos. Ramas verticiladas a fasciculadas, con surcos longitudinales y entrenudos de $0.5-10 \mathrm{~cm}$ de largo. Hojas reducidas, efímeras, no fotosintéticas, simples, opuestas y decusadas o dispuestas en verticilos de tres, las 
bases de las hojas connadas formando una vaina. Estróbilos compuestos, sésiles o cortamente pedunculados, situados en los nudos de ramas jóvenes. Estróbilos masculinos de 4-10 mm de largo; brácteas membranáceas o carnosas, organizadas en 2-8 pares opuestos o en 2-8 verticilos de tres, las inferiores estériles, las superiores con un esporangióforo de 1.9-5 mm de largo, portador de 2-10(-15) microsporangios biloculares, sésiles o cortamente estipitados, con dehiscencia poricida apical; polen elipsoidal acostillado o poliplicado, con 6-12 surcos paralelos al eje más largo. Estróbilos femeninos de 5-15 mm de largo; brácteas membranáceas o carnosas, organizadas en 2-10 pares opuestos o en 2-10 verticilos de 3, con uno o varios óvulos en la axila de las superiores, tegumento prolongado en el ápice por encima de las brácteas, formando un tubo micropilar, gametofito femenino con dos arquegonios. Semillas 1-3 en cada estróbilo, amarillas a pardas oscuras, lisas o rugosas, con 2 cotiledones. Con taninos y alcaloides, particularmente efedrina en algunas especies asiáticas. Número cromosómico base $\mathrm{x}=7$. Con poliploidía.

Ephedra se distribuye en zonas áridas y semiáridas de América, Asia, Europa y del norte de África; de Norteamérica se conocen 13 especies (Hollander et al., 2009) y de México ocho.

Clave para las especies de Ephedra de México

1 Hojas y brácteas generalmente en verticilos de tres.

2 Estróbilos siempre sésiles; semillas escabras, color pardo claro a verde amarillento; planta sólo conocida del noreste de Chihuahua ........... E. torreyana

2 Estróbilos usualmente con pedúnculos cortos, escamosos (rara vez sésiles); semillas usualmente lisas, color pardo oscuro o pardo claro.

3 Yemas terminales espinosas; vaina persistente y lacerada, volviéndose gris con la edad, papirácea; brácteas del estróbilo masculino pardo rojizas y las del estróbilo femenino translúcidas con centro pardo rojizo; semillas elípticas, más largas que anchas, pardas claras, 9-14 mm de largo ........ E. trifurca

3 Yemas terminales no espinosas; vaina subpersistente a completamente decidua, coriácea; brácteas del estróbilo masculino anaranjado amarillentas y las del estróbilo femenino amarillas pálidas con tintes anaranjados; semillas casi tan largas como anchas, casi globosas, color pardo claro a oscuro, 7-10 mm de largo ................................................. E. californica

1 Hojas y brácteas opuestas. 
4 Brácteas maduras de los estróbilos femeninos membranáceas, no carnosas.

5 Semillas solitarias, pardas claras a oscuras, 5-8 mm de largo; vainas persistentes, pardas oscuras, nudos abultados; tallos jóvenes extremadamente ásperos a lisos y glaucos ........................................................ E. aspera

5 Semillas en pares, pardas a casi negras, 6-9 mm de largo; vainas deciduas, grises; nudos no abultados; tallos jóvenes casi lisos o ligeramente ásperos E. nevadensis

4 Brácteas maduras de los estróbilos femeninos carnosas.

6 Semillas solitarias; estróbilos sésiles o subsésiles E. antisyphilitica

6 Semillas en pares; estróbilos sésiles a pedunculados.

7 Arbustos compactos, no trepadores; ramas rígidas; estróbilos femeninos sésiles o con pedúnculos de hasta $3 \mathrm{~mm}$ de largo; estróbilos masculinos con pedúnculos de hasta $1 \mathrm{~mm}$ de largo E. compacta

7 Arbustos trepadores; ramas laxas; estróbilos femeninos con pedúnculos de hasta $20 \mathrm{~mm}$ de largo; estróbilos masculinos con pedúnculos de hasta $12 \mathrm{~mm}$ de largo E. pedunculata

Ephedra antisyphilitica Berland. ex C.A. Mey., Mém. Acad. Imp. Sci. St.-Petersbourg, Sér. 6, Sci. Math. Seconde Pt. Sci. Nat. 5(2): 291. 1846. non Ephedra antisyphilitica S. Watson, nom. illeg. Tipo: Estados Unidos. Texas: près Rio Frio entre Laredo et [San Antonio de] Bejar, feb 1828, J.L. Berlandier 1590 (holotipo: MO! http:// www.tropicos. org/Image/100123591; isotipo: GH).

Ephedra occidentalis Torr. ex Parl., Prodr. DC. 16(2.2): 354. 1868. Tipo: no localizado.

Ephedra texana E.L. Reed, Bull. Torrey Bot. Club 62: 43. 1935. Tipo: Estados Unidos. Texas: Buffalo Springs, Lubbock, 15 abr 1934, E.L. Reed 3628 (holotipo: US! http://botany. si. edu/datasearch/types/showImage.cfm?mypic= 00012124.jpg).

Ephedra antisyphilitica Berland. ex C.A. Mey. var. brachycarpa Cory, Rhodora 40 (473): 218. 1940. Tipo: Estados Unidos. Texas: Eastern Bexar County, 25 mar 1935, H.B. Parks 12175 (holotipo: supuestamente en US).

Arbustos erectos o extendidos, 0.25-1 m de altura. Tallos jóvenes de color verde, volviéndose verde amarillentos y después verde grisáceos, casi lisos, con numerosos surcos longitudinales; la madera de tallos maduros cinérea, ligeramente fragmentada y fisurada. Ramas rígidas, duras, cilíndricas, de hasta $4 \mathrm{~mm}$ de grosor, alternas o verticiladas en los nudos, con ángulo de divergencia de aproximadamente 48 grados; entrenudos de $2-5 \mathrm{~cm}$ de largo. Yemas terminales 2-3 mm 
de largo, con ápice obtuso. Hojas opuestas, 1-3 mm de largo, con ápice obtuso que surge a partir de un engrosamiento herbáceo medio-dorsal, connadas en 2/3 a 9/10 de su longitud total, vaina membranácea, decidua. Estróbilos masculinos solitarios o en pares, en los nudos de las ramas jóvenes, lanceolados a elipsoides, 5-8 mm de largo; sésiles o subsésiles; brácteas 5-8 pares, opuestas, obovadas, connadas en 1/8 de su longitud desde la base, 2-3.5 mm de largo, 2-3 mm de ancho, ligeramente engrosadas, márgenes membranáceos, de color verde pálido a rojizo, el par inferior vacío; microsporangióforo 4-5 mm de largo, exerto en la mitad de su longitud, con 4-6 microsporangios sésiles o con un estípite muy corto. Estróbilos femeninos solitarios, en pares o en verticilos en los nudos de las ramas jóvenes, elipsoides, 6-11 mm de largo, casi sésiles; brácteas 4-6 pares, opuestas, ovadas, connadas en $1 / 8$ a $7 / 8$ de su longitud, los pares internos se vuelven carnosos, rojos y suculentos cuando maduros. Semillas solitarias, trígonas, ocasionalmente tetrágonas en corte transversal, de color pardo claro a oscuro, lisas, 6-9 mm de largo, 2-3.5 mm de ancho, conspicuamente exertas; pico micropilar recto, ligeramente exerto, limbo ligulado ligeramente contorto.

En la etiqueta original del ejemplar tipo se puede observar en el extremo superior izquierdo una nota que dice: número $1590=320$; es probable que el número 320 corresponda a otro ejemplar colectado por la misma persona, de igual especie.

Para Ephedra antisyphilitica var. brachycarpa Cory se indica en la descripción original que el holotipo se encuentra depositado en el herbario US, pero Cutler (1939) en su monografía afirma no haberlo visto. El ejemplar parece estar perdido.

Esta especie crece en suelos calizos, yesosos y rocosos, en matorral xerófilo, pastizal y dunas. Alt. 300-2350 m. Se distribuye en el suroeste y centro de Estados Unidos de América (Nuevo México, Oklahoma, Texas) hasta México (Aguascalientes, Baja California, Coahuila, Durango, Nuevo León, San Luis Potosí, Sonora y Tamaulipas). Fig. 1.

Nombres comunes: cañatilla, popotillo, tepopete.

Ejemplares examinados. Aguascalientes: Mpio. Asientos. Mina El Orito, $H$. Puig 6661 (MEXU). Baja California: Mpio. Ensenada. Lower Guadalupe Canyon, SW of Mexicali. Sierra de Juárez, D. Charlton 1318 (MEXU). Ensenada, Las Lagunitas, R. Moran 12703 (MEXU). Mpio. Tecate. Sierra Juárez, La Rumorosa, E. Ezcurra et al. 31 marzo 1989 (MEXU). Mpio. Indefinido. Sierra La Asamblea, 


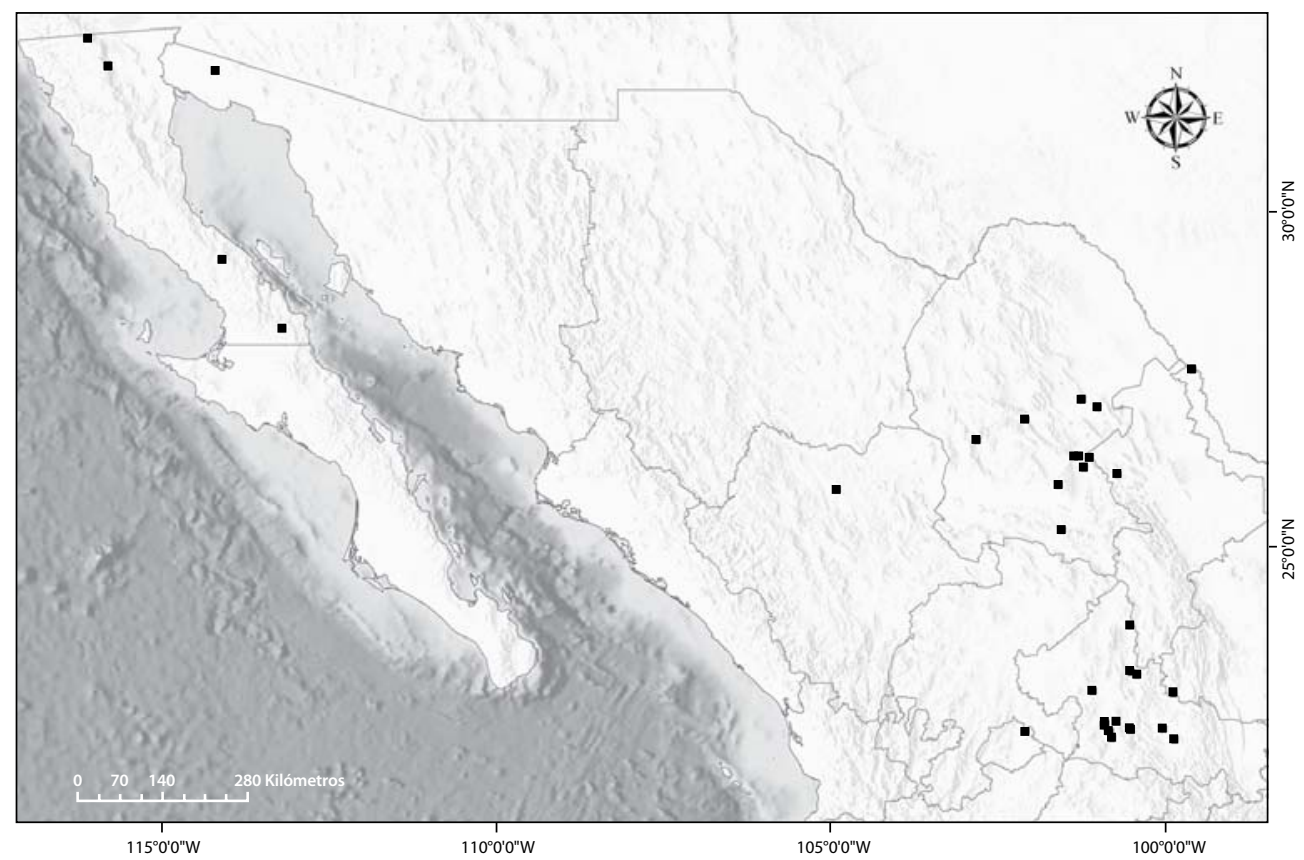

Fig. 1. Distribución de E. antisyphilitica en México.

heading in from el crucero of highway 1, 13.5 miles $\mathrm{N}$ of junction with main road to Bahía de los Ángeles, in cym SE side of cerro La Gobernadora, S. Boyd y T. Ross 5565 (MEXU). Coahuila: Mpio. Abasolo. Sierra de la Rata, M.C. Johnston et al. 10167 (MEXU). Mpio. Castaños. $4 \mathrm{~km}$ W of La presa de Chaires, M.C. Johnston et al. 10274 (MEXU). Mpio. Cuatro Ciénegas. Dunas cercanas a la poza de La Becerra en Cuatro Ciénegas, J.Á. Villarreal et al. 3197 (MEXU). Mpio. General Cepeda. Cañón de Loma Prieta, Sierra de la Paila, J. Marroquín 2327 (MEXU). Mpio. Ramos Arizpe. Transecto Santa Teresa - Santa Genoveva, partes bajas de Sierra de la Gavia, J.Á. Villarreal y M.A. Carranza 4060 (MEXU). Mpio. San Pedro. Canyon in E side of Sierra de las Margaritas about $13 \mathrm{~km}$ N of Las Margaritas, M.C. Johnston et al. 10359 (MEXU). Mpio. Indefinido. $2 \mathrm{~km} \mathrm{SW}$ of restaurant La Muralla, M.C. Johnston et al. 10287, 10288 (MEXU). Sierra de la Paila at mina La Abundancia, M.C. Johnston et al. 10519 (MEXU). Entre Saltillo y Monclova, J. Valdés y F. Miranda A-119 (MEXU). Durango: Mpio. Hidalgo. 11 miles E of La Zarca, route number 30, D.S. Correll y I.M. Johnston 20234 (MEXU). Nuevo León: Mpio. Mina. Minas Manto Blanco y Sábana Blanca, just N of the Cañón de Potrerillos, M.C. Johnston et al. 10251B (MEXU). San Luis Potosí: Mpio. Guadalcázar. San José del Refugio, km 550 carretera central, 
entre entronque y Matehuala, F. Medellín 1136 (ENCB). Mpio. Rioverde. $20 \mathrm{~km}$ del entronque Matehuala - Dr. Arroyo, C. Méndez 52 (SLPM). Mpio. San Luis Potosí. Jassos, por la carretera que va hacia Río Verde, J. García 25 junio 1978 (SLPM). 15 km al N-NE de la ciudad de San Luis Potosí, J. Rzedowski 3540 (ENCB). 3 km al NE de Peñasco, S. Salas 404 (SLPM). Mpio. San Nicolás Tolentino. 4 km NO del rancho Juan Sarabia, R. Banda 6 octubre 1973 (MEXU). San Nicolás Tolentino, F. Medellín 3 julio 1965 (MEXU, SLPM). Mpio. Soledad de Graciano Sánchez. Palma de la Cruz, A. Gómez 830 (ENCB, SLPM), 11354 (MEXU). La Joya, 4 km al NO de Ventura, J. Rzedowski 7539 (ENCB). Mpio. Tanque de los Caballos. Km 33 carretera San Luis Potosí - Antiguo Morelos, F. Medellín 861 (SLPM). Mpio. Venado. San Rafael, NO Moctezuma, A. Gómez 643 (ENCB, MEXU). Mpio. Villa de Guadalupe. Km 555 carretera México - Piedras Negras, cerca de Vallejo, J. Rzedowski 9170 (ENCB). 10 km después del entronque de carretera a San Nicolás Tolentino con la carretera San Luis Potosí - Matehuala, ejido Presita de la Cruz, L. Villanueva y R.M. Fonseca 1 (FCME). Km 144 de la carretera 57 San Luis Potosí - Matehuala, en lado oeste, L. Villanueva y R.M. Fonseca 4 (FCME). Mpio. Indefinido. Camino de terracería El Cedral - Salado (20 km N de Rancho Nuevo), H. Flores 183 (MEXU). Km 623 carretera central, San Luis Potosí - Saltillo, A. Gómez 310 (MEXU), 810 (MEXU). Santa Rosa, F. Medellín 1378 (SLPM). La Muralla, F. Takaki 15 noviembre 1967 (SLPM). Sonora: Mpio. San Luis Río Colorado. Sierra del Rosario, Gran Desierto, N side of main mountain mass, R.S. Felger et al. 75-14A (MEXU). Tamaulipas: Mpio. Tula. $15 \mathrm{~km}$ al N del límite de Nuevo León y Tamaulipas, F. González-Medrano et al. 2000 (MEXU). $32 \mathrm{~km}$ al SO de Tula, cerca del límite de Tamaulipas con San Luis Potosí, F. González-Medrano et al. 4355 (MEXU).

Ephedra aspera Engelm. ex S. Watson, Proc. Amer. Acad. Arts 18: 157. 1883. Ephedra nevadensis var. aspera (Engelm. ex S. Watson) L.D. Benson, Amer. J. Bot. 30(3): 232. 1943. Tipo: México. Coahuila: Sierra Madre, 40 miles south of Saltillo, jul 1880, E. Palmer 1288 (lectotipo: MO! http://www.tropicos.org/Image/45632, designado por Cutler, 1939; isolectotipos: NY! US! GH!).

Ephedra peninsularis I.M. Johnst., Univ. Calif. Publ. Bot. 7: 437. 1922. Tipo: México. Baja California: Magdalena Island, Lower California, 12 ene 1889, T.S. Brandegee s.n. (holotipo: probablemente UC; isotipo: GH! código de barras 22603).

Ephedra reedii Cory, Rhodora 40(473): 216. 1938. Tipo: Estados Unidos. Texas: Brewster County, 55.4 miles south of Alpine on the road to Terlingua, 13 abr 1936 V.L. Cory 18547 (lectotipo: GH! código de barras 22599, designado por Cutler, 1939). 
Arbustos erectos, 0.25-1.25 m de altura. Tallos jóvenes de color verde pálido a verde oscuro, volviéndose amarillos con la edad, de extremadamente ásperos a lisos y glaucos, con numerosos surcos longitudinales; la madera de tallos maduros cinérea, fragmentada y fisurada. Ramas rígidas, firmes, cilíndricas, de hasta 3 $\mathrm{mm}$ de grosor, opuestas o verticiladas en los nudos, con ángulo de divergencia de aproximadamente 35 grados; entrenudos de $1.5-5 \mathrm{~cm}$ de largo. Yemas terminales de 1-2 mm de largo, cónicas, con ápice obtuso. Hojas opuestas, 1-2.5 mm de largo, con ápice obtuso que surge a partir de un engrosamiento herbáceo medio-dorsal, connadas en 1/2-7/8 de su longitud total, vaina persistente. Estróbilos masculinos en pares, rara vez solitarios o verticilados en los nudos de las ramas jóvenes, obovoides, 4-7 mm de largo; sésiles o rara vez cortamente pedunculados; brácteas 6-10 pares, opuestas, obovadas, $3 \mathrm{~mm}$ de largo, $2 \mathrm{~mm}$ de ancho, membranáceas, de color amarillo a pardo rojizo, el verticilo inferior vacío; microsporangióforo 4-5 $\mathrm{mm}$ de largo, exerto en 1/3 de su longitud, con 4-6 microsporangios sésiles o con un estípite muy corto. Estróbilos femeninos en pares, ocasionalmente solitarios o en verticilos en los nudos de las ramas jóvenes, ovoides, 6-10 mm de largo; sésiles o con un pedúnculo corto y escamoso; brácteas 5-7 pares, opuestas, orbiculares, 2-5 $\mathrm{mm}$ de largo, 2-5 $\mathrm{mm}$ de ancho, engrosadas, color pardo rojizo, márgenes membranáceos. Semillas solitarias, en corte transversal de forma circular a ligeramente trígonas, de color pardo claro a oscuro, lisas a ligeramente ásperas, 5-8 mm de largo, 2.5-4 mm de ancho, excediendo en 1/3 el tamaño de las brácteas; pico micropilar ligeramente exerto, casi recto, limbo contorto.

Crece en terrenos calizos, yesosos y rocosos, en matorral xerófilo, pastizal y vegetación cercana a la costa. Alt. 280-2300 m. Se distribuye del SO de Estados Unidos de América (Arizona, California, Nuevo México, Texas) a México (Baja California, Baja California Sur, Chihuahua, Coahuila, Durango, Hidalgo, Nuevo León, San Luis Potosí, Sonora, Tamaulipas y Zacatecas). Fig. 2.

Nombres comunes: cañutilla, hintimoreal, ítamo real, pitamoreal, popotillo, tepopote.

Ejemplares examinados. Baja California: Mpio. Ensenada. Isla Cedros, A.W. Anthony 281 (MEXU). Small sierra E of La Ramona on road from Guayaquil to puerto Santa Catarina, NW of Catavina, S. Boyd y T. Rose 5368 (MEXU). 10-20 miles $\mathrm{N}$ of puerto Santa Catarina on road to rancho Santa Catarina, D.E. Breedlove 62270 (MEXU). Canyon of the Sierra de Placeros, above Puerto Nuevo between 


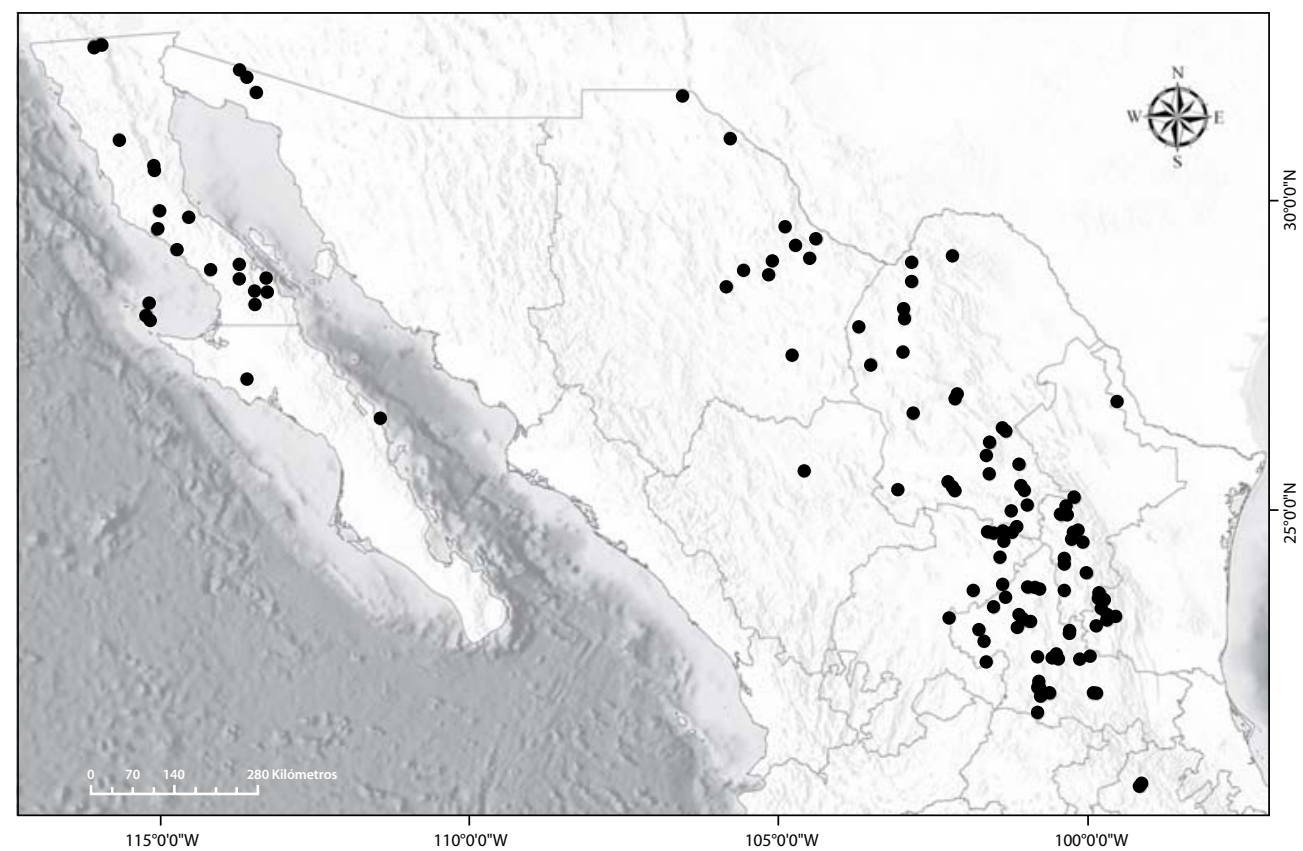

Fig. 2. Distribución de E. aspera en México.

Bahía Asención and Bahía Tortugas, D.E. Breedlove 62401 (MEXU). 15 km inland from Punta Canoas on road to Cataviña, D.E. Breedlove 72845 (MEXU). 1.9 km S, $1.3 \mathrm{~km}$ W of Los Tepetates, S of Sierra de Calmalli, T.L. Burgess et al. 6461 (MEXU). Mesa top $0.1 \mathrm{~km} \mathrm{N,} 1.1 \mathrm{~km}$ E of San Borja Mission, T. L. Burgess y K. Cole 6502 (MEXU). Isla Cedros, Los Quiotes, H. Cota 7400 (ENCB). $30 \mathrm{~km}$ al N de Santa Rosalía, C.L. Díaz 4128 (MEXU). Isla Cedros, G. Fleming 1 agosto 1931 (MEXU). Isla Cedros, F. Giovanini 6 febrero 1976 (ENCB). Isla Cedros, W of the village 0.1 miles, A.L. Haines y G. Hale 17 febrero 1939 (MEXU). Mouth of valley of San Juan, Sierra San Borja, R. Moran 8498 (ENCB). Cerro Santa María, R. Moran 11472 (ENCB). Cima del Cerro Potrero, R. Moran 12161 (ENCB). Boca del Arroyo. 7 miles NW de Punta Blanca, R. Moran 17129 (ENCB, MEXU). Cañón El Cajón, rancho El Cajón, base of Sierra San Pedro Mártir, San Felipe Desert, W of San Felipe, R. Puente y G. Reinhardt 552 (ENCB, SLPM). Isla Cedros, Cañada de la Mina, 1.5 miles from $\mathrm{N}$ end of island, to top of pine ridge, R.F. Thorne 58561 (MEXU). Above $\mathrm{S}$ end of Bahía de Los Ángeles, G.L. Webster 21502 (MEXU). 12.5 miles NE of Mission San Borja, G.L. Webster 21533 (MEXU). 10 miles W of Bahía de Los Ángeles, I.L. Wiggins y D.B. Wiggins 16017 (MEXU). Mpio. Tecate. $7 \mathrm{~km}$ al E del poblado de La 
Rumorosa, carretera Mexicali - Tijuana, H. Flores et al. 269 (ENCB, MEXU). Isla Cedros, L.M. Villarreal 10667 (ENCB). Baja California Sur: Mpio. Loreto. S side of punta El Pulpito, I.L. Wiggins 17438 (MEXU). Mpio. Mulegé. Picachos de Santa Clara, R. Moran y J.L. Reveal 19709 (ENCB). Chihuahua: Mpio. Aldama. 24 km NW of Potrero which is on the Río Conchos, near the NW end of the Sierra Cuesta del Infierno, F. Chiang et al. 8375D (MEXU). Sierra Chorreras, low (25 m) ridge along SE side of main arroyo of Cañón Pedregoso, N of $2150 \mathrm{~m}$ SW peak of range, 8.3 miles E of rancho El Tanque and nearly 6 air miles ESE of Chorreras, T. Wendt $y$ E.J. Lott 721A (MEXU). Bluffs along Arroyo Nogales 2.4 miles S Falomir railroad bridge (Río Conchos, 0.2 mi NE railroad), T. Wendt y E.J. Lott 767A (MEXU). Mpio. Aquiles Serdán. Santa Eulalia Mountain, M.V. García Miranda y M.T. Germán 4 junio 1985 (MEXU). Mpio. Camargo. $11 \mathrm{~km} \mathrm{~W}$ of headquarters of Las Pampas on the road to Camargo, M.C. Johnston et al. 10531 (MEXU). Mpio. Coyame del Sotol. $1.5 \mathrm{~km}$ SE of rancho Boquillas Pérez on the road towards San Luis (which is on the Ojinaga - La Perla highway), M.C. Johnston et al. 10555 (MEXU). NE side of Sierra Cuchillo Parado, M.C. Johnston et al. 10580 (MEXU). Mpio. Guadalupe. $21 \mathrm{~km}$ al S de Cedillos, G. Borja B-387 (MEXU). Mpio. Juárez. Sierra Juárez on W side of Ciudad Juárez, nearly $6 \mathrm{~km}$ S of US-Mexico border, R. Spellenberg y J. Bacon 10980 (MEXU). Mpio. Ojinaga. $11 \mathrm{~km} \mathrm{~S}$ of San Luis on the Ojinaga - La Perla highway, M.C. Johnston et al. 10550 (MEXU). $17 \mathrm{~km} \mathrm{~S} \mathrm{of} \mathrm{Ojinaga} \mathrm{on} \mathrm{the} \mathrm{highway} \mathrm{to} \mathrm{La} \mathrm{Perla,}$ M.C. Johnston et al. 10743 (MEXU). Coahuila: Mpio. Acuña. Camino Palmas Altas - La Victoria, H. Mendiola 1 marzo 1961 (ENCB). Camino Palmas Altas - La Victoria, A. Salgado 1 marzo 1961 (ENCB). Mpio. Castaños. $2 \mathrm{~km} \mathrm{SW}$ of restaurant La Muralla, M.C. Johnston et al. 10289 (MEXU). Mpio. Cuatro Ciénegas. Sierra de San Marcos, low north end SW of Cuatro Ciénegas, M.C. Johnston et al. 10917C (MEXU). Tip of Sierra de San Marcos, D. J. Pinkava et al. 5134 (ENCB). Mpio. General Cepeda. $195 \mathrm{~km}$ E of Torreón, on the way from Saltillo to Torreón, alongside of highway 45D, T. Yahara et al. 725 (MEXU). Mpio. Ocampo. Minas San Francisco, $160 \mathrm{~km}$ al NO de Muzquiz, F. Brizuela 755 (ENCB). Sierra del Pino, ejido Acebuches, cañón La Vaca, M.A. Carranza et al. C-952 (MEXU). S side of cerro San José de las Piedras, F. Chiang et al. 7556 (MEXU). Western base of Picacho del Fuste, northeasterly from Tanque Vayonetta, I.M. Johnston 8452 (MEXU). Sierra Santa Fe del Pino, broad canyon between the two main ridges, $\mathrm{W}$ of mina La Pasión, M.C. Johnston et al. 11263 (ENCB). Mpio. Parras. Sierra de Parras, nearly 9 km S of Parras towards San Francisco del Barrial, C.D. Bailey 36 (MEXU). Ejido Los Hoyos, Sierra de Parras, M.A. Capó 1 febrero 1978 (MEXU). Sierra de Parras, 2.4 km (1.5 millas) al $\mathrm{S}$ del Ejido Colorado, $8.8 \mathrm{~km}(5.5 \mathrm{mi})$ al O de Parras, C.P. Cowan y A. Rodríguez 
3632 (MEXU). Sierra de Parras en el Cañón de Gustrola, A. Rodríguez y P. Antonio Hernández 1 septiembre 1981 (ENCB). Ejido Charco de la Vaca, aproximadamente $12 \mathrm{~km}$ al S de Parras de la Fuente, A. Rodríguez y M.A. Carranza 765 (MEXU). Cañón de Gustrola en la Sierra de Parras, A. Rodríguez y M. Martínez 10 octubre 1980 (MEXU). Mpio. Ramos Arizpe. In upper reaches of cañada El Aguirreño, from 0-1 km W of old barita mine, nearly 4-5 air km from mouth of canyon, nearly 11 air km SE of Las Coloradas, on NW side of sierra La Paila, Las Coloradas, E.L. Bridges et al. 13064 (MEXU). Sierra La Paila at one of the lower mine levels in the mina La Abundancia area, M.C. Johnston et al. 10512 (MEXU). La cuesta de Palmas Altas, Sierra del Sombrero, J. Passini y M.F. Robert 5726 (ENCB). NW side of sierra La Paila, cañada El Aguirreño, along gravel road to old barite mine, L. Woodruff et al. 315 (MEXU). San Lázaro, F.L. Wynd y C.H. Mueller 143 (MEXU). Mpio. Saltillo. Rancho Los Ángeles, $48 \mathrm{~km}$ al S de Saltillo, J.M. Ávila, abril 1980 (ENCB). Puerto de Rocamontes, J.Á. de la Cruz 28 febrero 1961 (ENCB). Near Saltillo, G.B. Hinton 16664 (ENCB). Puerto de Rocamontes, at the Zacatecas - Coahuila state line, M.C. Johnston et al. 10494B (MEXU). About $6 \mathrm{~km}$ airline W of Saltillo, E extremity of Sierra de la Vega, at end below estación microondas La Vega, M.C. Johnston et al. 10502 (MEXU), 10503 (MEXU). In arroyo, $3 \mathrm{~km} \mathrm{SW}$ of Fraile, L.R. Stanford et al. 338 (MEXU). Mpio. San Pedro. Canyon in E side of sierra de Las Margaritas about $13 \mathrm{~km} \mathrm{~N}$ of Las Margaritas, M.C. Johnston et al. 10365 (MEXU). Mpio. Sierra Mojada. Puerto de Flores, sobre la carretera central a Coahuila, A. Gómez 628 (MEXU). Vicinity of Santa Elena mines, eastern foothills of the Sierra de las Cruces, R.M. Stewart 2228 (MEXU). Mpio. Viesca. Sierra de Parras, aproximadamente 3 km al SO de Ojo de Agua, A. Rodríguez 30 enero 1981 (SLPM). Mpio. Indefinido. Camino San Juan de Mimbres, Ciénega del Toro, J. Elizondo y R. Banda 268 (MEXU). Durango: Mpio. San Pedro del Gallo. Loma ancha del pueblo Cieneguilla, O. García 80 (ENCB). Hidalgo: Mpio. Cardonal. Cardonal, $18 \mathrm{~km}$ al NE de Ixmiquilpan, $L$. González 2260A (ENCB). Mpio. Ixmiquilpan. Sabanilla, $14 \mathrm{~km}$ al NE de Ixmiquilpan, L. González 2260B (ENCB). Nuevo León: Mpio. Aramberri. 41 km NNE of Dr. Arroyo, on the way from Matehuala to Aramberi, T. Kajita et al. 98110603 (MEXU). Mpio. Dr. Arroyo. 39 miles S of San Roberto (junction of Galeana road), on the Saltillo - Matehuala highway, J. Crutchfield y M.C. Johnston 6039 (MEXU). 50 km al S de José de Raíces, sobre la carretera a Matehuala, J. Rzedowski 32277A (ENCB). Mpio. Galeana. $2 \mathrm{~km}$ al E de las Casas, ejido El Tokio, O. Briones y J. Treviño OB463 (ENCB). Sierra Madre Oriental, between Arena and Valle Hermosa, about $60 \mathrm{~km} \mathrm{NE}$ of Dr. Arroyo, Z. Debreczy et al. 38986b (MEXU). El Potosí, SW of Galeana, G.H. Hinton et al. 18508 (MEXU). La Becerra, G.H. Hinton et al. 19672 (MEXU). Plains, 
hills, and cañons above ranchito San Enrique, hacienda San José de Raíces, C.H. Mueller 2353 (MEXU). Edge of road 31 miles N of San Roberto, route 57, D. Siegler et al. 13742 (MEXU). Aproximadamente $7 \mathrm{~km}$ de San Roberto, rumbo a Galeana, en el km 87, J. Valdés y M.A. Carranza VR-2536 (ENCB). Áreas cercanas a Pablillo, aproximadamente $20 \mathrm{~km}$ al S de Galeana, J.Á. Villarreal et al. 3023 (ENCB, MEXU). Mpio. Rayones. Las Trancas, C.H. Mueller 2157 (MEXU). Mpio. Indefinido. Carretera central km 681, 13 km al N de Santa Ana, F. Medellín 945 (ENCB). San Luis Potosí: Mpio. Catorce. Km 14 brecha Cedral - Catorce, C.L. Díaz 3717 (ENCB). Alrededores de la mina de la Cocona, A. Gómez-González 743 (ENCB, MEXU). 17.5 km from (roughly SW) of Cedral on the carretera to Real de Catorce, M.C. Johnston et al. 11064 (MEXU). Mpio. Cedral. Cerro al SO de Cedral, A. Gómez 948 (ENCB, MEXU, SLPM). Mpio. Cerro de San Pedro. 3 km al E de Jassos, J. Ballín 535 (SLPM). Mpio. Charcas. Charcas, C.L. Lundell 5165 (MEXU). Aproximadamente $6 \mathrm{~km}$ al E de Laguna Seca, km 20 carretera San Luis - Antigua Morelos, J. Rzedowski 5791 (ENCB). Aproximadamente $12 \mathrm{~km}$ al O de la estación Berrendo, J. Rzedowski 6591 (ENCB, MEXU). Estación Laguna Seca, S. Salas 8 noviembre 1987 (SLPM). Mpio. Ciudad del Maíz. Charco Blanco, camino a Guadalcázar, S. Fuentes 27 noviembre 1955 (MEXU). Tepeyac y Cardonal, A. Gómez 615 (SLPM). Mpio. Guadalcázar. 1 $\mathrm{km}$ de la desviación a Guadalcázar, N.C. Cárdenas y J. García 9 (SLPM). Aproximadamente $3 \mathrm{~km}$ al NO de Charco Blanco, F. Medellín 1552 (ENCB, MEXU), 1553 (SLPM). 4 km al O de Charco Blanco, km 76 carretera S.L.P. - Antigua Morelos, J. Rzedowski 6740 (SLPM). Santa Rita del Rucio, La Puentecita, 2.5 km al NE de San Carlos, ejido Santa Rita del Rucio, $R$. Torres RTC14784 (MEXU, SLPM). El Cañón Hondo, $12 \mathrm{~km}$ al NO de Guadalcázar, hacia el crucero S.L.P - Matehuala, R. Torres 15601 (MEXU). Mpio. Rioverde. Entre Tecomate y Salinas. Carretera San Luis Potosí - Salinas, A. Gómez 734 (SLPM). Camino Rioverde - Cárdenas, vía San Francisco, J. Rzedowski 5143 (SLPM). Mpio. San Luis Potosí. 2 km de Jassos, por la carretera que va hacia Rioverde, C. Méndez 76 (SLPM). Mpio. Santa María del Río. Cerro La Calera del Trinquete a Pedreras, A. Gómez 636 (MEXU). Mpio. Santo Domingo. Rancho Guadalupe, entrada N, km 20 del camino que va hacia San Francisco, C. Méndez 61 (MEXU, SLPM). Por el camino Rancho de Guadalupe hacia San Francisco, C. Méndez 62 (SLPM). 4 km al O del Bozal por el camino hacia la Sierra, C. Méndez 65 (SLPM). Mpio. Soledad de Graciano Sánchez. Frente al panteón de Cerro de San Pedro, N.V. Barragán 2 (MEXU, SLPM). Mpio. Villa de Arista. 5 $\mathrm{km}$ al E de Villa de Arista, R. Aguirre 11 junio 1968 (ENCB). Mpio. Villa de Ramos, $20 \mathrm{~km}$ al NE de Punteros, P. Castillo 4 octubre 1997 (SLPM). $7 \mathrm{~km}$ al N de la Herradura, A. Gómez 764 (SLPM). Mpio. Zaragoza. San Francisco del Refugio - 
Palula, A. Gómez 658 (MEXU, SLPM). 17 km de la ciudad de San Luis Potosí, carretera a Rioverde, S. Salas 501 (SLPM). $1.5 \mathrm{~km}$ al O de la Esperanza, S. Salas 16 junio 1993 (SLPM). Mpio. Indefinido. Km 685 de la carretera central, A. Gómez 817 (MEXU). $2.3 \mathrm{~km}$ del entronque Matehuala - Dr. Arroyo al E del camino a ejido La Chiripa, C. Méndez 56 (ENCB). Al E del Rancho Palula, C. Méndez 63 (SLPM). A la altura de las faldas SE del cerro del Tunalillo, J.A. Reyes 1026 (SLPM). Km 20 carretera San Luis - Rioverde, J. Rzedowski 6168 (MEXU). 1 km después del entronque hacia Guadalcázar, L. Villanueva y R.M. Fonseca 2 (FCME). Sonora: Mpio. Puerto Peñasco. $20 \mathrm{~km}$ del cráter Mc. Dougal, región del Pinacate, E. Ezcurra et al. 15 abril 1981 (MEXU). Mpio. San Luis Río Colorado. Upper N slope of the Hornaday Mountains (= sierrita El Temporal), near the W end, NW of Sierra Pinacate, T.L, Burgess y R.M. Turner 6834 (MEXU). Piedemonte al NO del Pinacate, E. Ezcurra et al. 29 abril 1981 (MEXU). Mpio. Indefinido. Sierra El Rosario, in Gran Desierto near end of northern most ridge, SE of large star dune, T.L. Burgess y R.M. Turner 6873 (MEXU). Tamaulipas: Mpio. Bustamante. $2 \mathrm{~km}$ al SE de la Perdida, F. González-Medrano et al. 10320 (MEXU). 3 km O sobre la desviación a Bustamante carretera Tula - Ciudad Victoria, ejido El Capulín, M. Martínez 429 (ENCB, MEXU, SLPM). Mpio. Guerrero. $20 \mathrm{~km}$ al O de Ciudad Guerrero, F. González-Medrano et al. 6319 (MEXU). Mpio. Miquihuana. Rincón de las Vacas, 7 km al NO de Miquihuana entre Miquihuana y La Perdida, F. González-Medrano et al. 7018 (MEXU). 8 km al $\mathrm{N}$ de La Perdida, F. González-Medrano et al. 8646 (MEXU). $15 \mathrm{~km}$ al N de La Perdida, F. González-Medrano et al. 8740 (MEXU). $20 \mathrm{~km}$ al N de La Perdida, F. González-Medrano y P. Hiriart 13199 (MEXU). 4 miles W of Miquihuana near a large Tanque, J. Henrickson y V. Heuvel 22435 (MEXU). $2 \mathrm{~km}$ al S de Miquihuana, L. Hernández 1625A (ENCB, MEXU). 3 km al SO de Miquihuana rumbo al cañón del Soldado, J. Jiménez 101 (MEXU, SLPM). $1 \mathrm{~km}$ adelante de Miquihuana, $H$. Sánchez-Mejorada 3646 (MEXU). Mpio. Palmillas. Palmillas, M. Sánchez 14 septiembre 1942 (ENCB). Mpio. Tula. $25 \mathrm{~km}$ al N de Tula, F. González-Medrano et al. 168 (MEXU). 4 km al O de El Capulín y a 25 km al N de Tula, F. GonzálezMedrano 13239 (MEXU). $4 \mathrm{~km}$ al O de ejido El Salitrillo, P. Hiriart et al. 850 (MEXU), 854 (MEXU). Mpio. Indefinido. $16 \mathrm{~km}$ al E de Guadalcázar, San Luis Potosí, P. Hiriart et al. 490 (MEXU). Zacatecas: Mpio. Mazapil. Carretera Zacatecas - Saltillo, $10 \mathrm{~km}$ al NE del entronque San Tiburcio - Zacatecas, C.L. Fuentes 43 (MEXU). $2.5 \mathrm{~km}$ E of Mazapil on highway to Concepción del Oro, M.C. Johnston et al. 11545 (MEXU), 11545A (MEXU). El Durazno, $3 \mathrm{~km}$ del poblado por el camino a la Sierra de el Socavón, M.A. Juárez 78 (MEXU). Mpio. Indefinido. Km 199 de la carretera Zacatecas - Saltillo, J. García 310 (ENCB). Villa de Cos, $5.5 \mathrm{~km} \mathrm{~W}$ of the 
highest peak in the Sierra Sarteneja; $8 \mathrm{~km}$ E of Puerto Madero, M.C. Johnston et al. 10426 (MEXU). Camino al nivel de pabellón, en dirección de las minas, M.F. Robert y J. Passini 5883 (MEXU). Valle de El Rodeo, R. Sánchez y S. Cruz 1 julio 1972 (MEXU).

Ephedra californica S. Watson, Proc. Amer. Acad. Arts 14: 300. 1879. Tipo: Estados Unidos. California: San Diego County, Jamul Valley, 25 jun 1875, E. Palmer 364 (lectotipo: GH! código de barras 22596, designado por Cutler, 1939; isolectotipo: GH!).

Arbustos erectos o trepadores, 0.3-1 m de altura. Tallos jóvenes de color verde amarillento, volviéndose amarillos y después pardo amarillentos, a glaucos, con numerosos y tenues surcos longitudinales, la madera de tallos maduros de color café grisáceo, fragmentada e irregularmente fisurada. Ramas semiflexibles a rígidas, firmes, cilíndricas, de hasta $4 \mathrm{~mm}$ de grosor, solitarias o verticiladas en los nudos, con ángulo de divergencia de aproximadamente 45 grados; entrenudos 3-6 cm de largo. Yemas terminales, 2-3 mm de largo, con ápice agudo, cónicas no espinosas. Hojas dispuestas en verticilos de tres, 2-6 mm de largo, con ápice agudo a obtuso que surge a partir de un engrosamiento medio-dorsal, connadas en 1/2-3/4 de su longitud total; vaina subpersistente a completamente decidua. Estróbilos masculinos solitarios o varios en un verticilo en los nudos de ramas jóvenes, ovoides, 6-7.5 mm de largo corto pedunculados, pedúnculos escamosos; brácteas 8-12 en verticilos de 3, ovadas, ligeramente connadas en la base, 2.5-3 mm de largo y de ancho, membranáceas, de color naranja amarillento excepto en los márgenes hialinos, los verticilos inferiores vacíos; microsporangióforo 3-5 mm de largo, exerto en 1/3, con 3-7 microsporangios sésiles o corto-estipitados. Estróbilos femeninos solitarios o varios en verticilos de ramas jóvenes, ovoides, 7-10 mm de largo; cortamente pedunculados, pedúnculo escamoso; brácteas 4-6 en verticilos de 3, orbiculares, ligeramente unguiculadas, 5-7 mm de largo, 5-10 mm de ancho, color amarillo pálido excepto en las regiones basal y central que son de color naranja o naranja amarillento, márgenes enteros. Semillas solitarias, rara vez en pares, casi globosas pero ligeramente tetragonales en corte transversal, de color pardo claro a oscuro, lisas, 7-10 mm de largo y de ancho, igualando o excediendo en tamaño a las brácteas subyacentes; pico micropilar recto, ligeramente exerto, limbo ligeramente contorto y lígula pequeña.

Crece en terrenos arenosos, en matorral xerófilo, pastizal y dunas. Alt. 0-2100 m. Se extiende desde Estados Unidos de América (California, Arizona) hasta Méxi- 
co (Baja California, Baja California Sur). Se ha reportado su uso como remedio contra padecimientos renales. Fig. 3.

Nombres comunes: no conocidos.

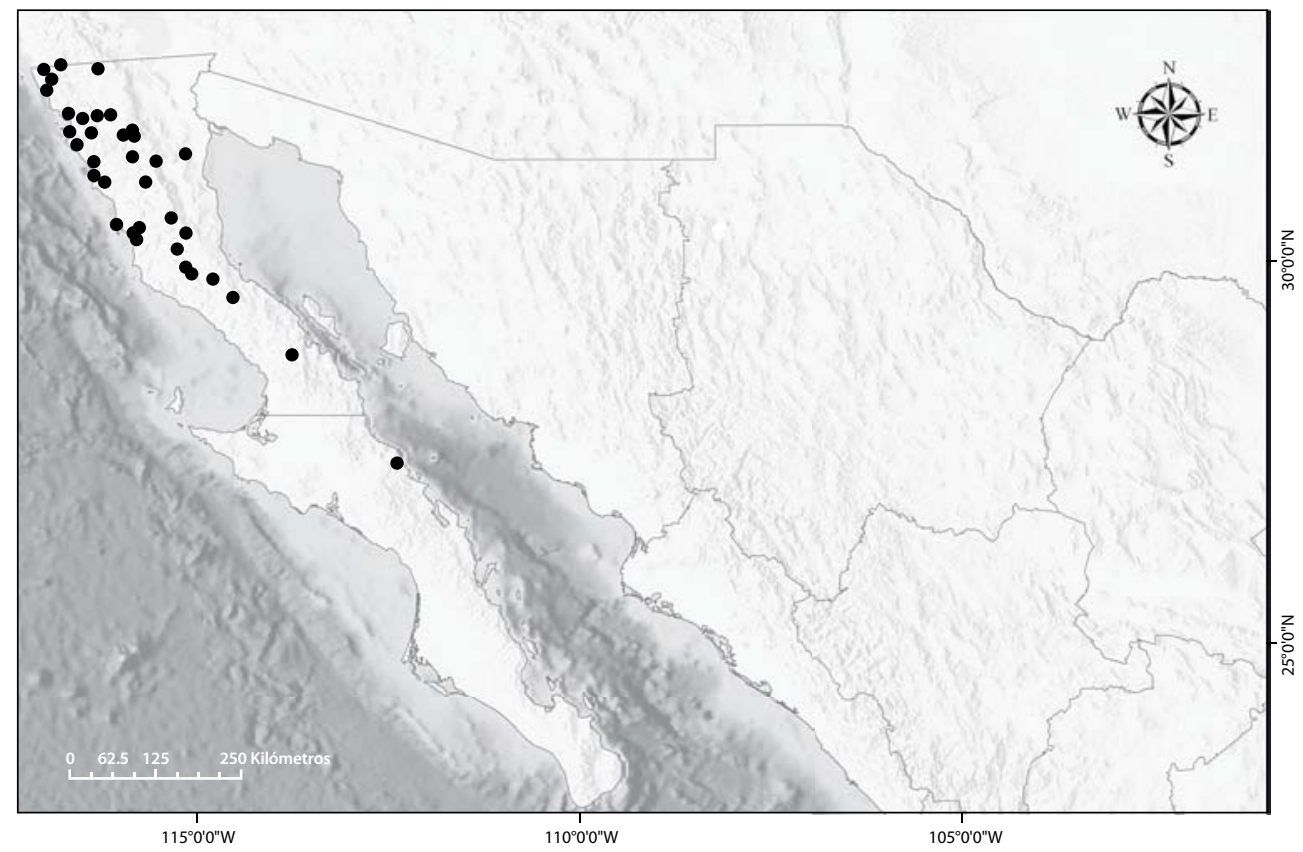

Fig. 3. Distribución de E. californica en México.

Ejemplares examinados. Baja California: Mpio. Ensenada. Small sierra E of La Ramona on road from Guayaquil to puerto Santa Catarina, NW of Catavina, S. Boyd y T. Ross 5369 (MEXU). Santa Inés study site, 9 km NW of rancho Santa Inés, sandy soil near Highway 1, R.D. Bratz 3104 (MEXU). 5 km NE of San Borja on road to Bahía de los Ángeles, D.E. Breedlove 60985 (MEXU). NW facing slope of Cerro Colorado in Cañón de Río San Ysidro, 6.6 miles SW of San Vicente, R.E. Broder 210 (MEXU). In canyon between village of Santa Catarina and lower dam, 64 miles SE of Ensenada, R.E. Broder 579 (MEXU). At N boundary of Paipai Reservation, along road to rancho La Ciénega, 3.5 miles $\mathrm{N}$ of Santa Catarina, 64 miles SE of Ensenada, R.E. Broder 788 (MEXU). 2 miles E of Santa Catarina, 64 miles SE of Ensenada, R.E. Broder 846 (MEXU). Vicinity of Ensenada, Todos Santos 
Bay, H.S. Gentry 7914 (ENCB). Rancho Ernesto Ramonetti, en el km 51 de la carretera Ensenada - San Felipe, ejido Real del Castillo Nuevo. Delegación El Álamo, L.E. López, 32 (MEXU). $5 \mathrm{~km} \mathrm{~S}$ of San Quintin along highway 1, M. Merello y D. Brunner 251 (MEXU). 20 miles S of San Quintin on dirt road E of Mexican Highway 1, S. Miller et al. 7383 (MEXU). NW of San Vicente, 7 miles, R. Moran 1975 (MEXU). Plain 2 miles $\mathrm{N}$ of Llano Colorado, R. Moran 13875 (MEXU). 9 road miles SE of El Progreso, R. Moran 16894 (ENCB). Rancho Cerro Prieto, R. Moran 18104 (ENCB). At summit of Cerro Matomí, R. Moran 20757 (MEXU). 2 miles N-NE of Punta San José, R. Moran 22521 (ENCB). 1.7 miles N of La Joya, Punta Banda, $R$. Moran 22802 (ENCB). 25 miles down wide wash opposite store at El Arenoso, D.M. Porter 158 (MEXU). Rancho El Coyote, $60 \mathrm{~km}$ al O de El observatorio de San Pedro Mártir, P. Tenorio 13263 (MEXU). S of San Antonio del Mar, 6.8 miles WNW of colonet on hwy 1, R.F. Thorne y D. Charlton 58799 (MEXU). Playas de San Quintín, just N of base of sand spit, R.F. Thorne y D. Charlton 58891 (MEXU). Head of San Matías pass, R.F. Thorne 60154 (ENCB), 60155 (ENCB). Rancho La Choya, W foothills of la Sierra de Juárez, near $3 \mathrm{~km}$ E-NE of rancho near El Bashisha, R.F. Thorne et al. 62422 (ENCB). Ejido San Juan de Dios, Sierra de Juárez, J. Villa 21 (ENCB). Valle de la Trinidad, J. Villa 101 (ENCB). Near 4 miles NW of Cataviña, G.L. Webster 21742 (MEXU). About 2 miles N of arroyo El Socorro near the road and about 1 mile inland from the ocean, between San Quintín and El Rosario, I.L. Wiggins y J.H. Thomas 15 (MEXU). Lower part of San Matías pass, 31 miles W of San Felipe highway, I.L. Wiggins y D.B. Wiggins 16046 (MEXU). 16 miles $\mathrm{N}$ of Camalú and about 0.5 mile W of highway number 1 , I.L. Wiggins 21534 (ENCB). Mpio. Mexicali. Camino a Ensenada, cerca del paraje Aguaje del Sapo aproximadamente $10 \mathrm{~km}$ del Parque Nacional de la Sierra de Juárez, A. Manzera AM-231 (ENCB, SLPM). Mpio. Playas de Rosarito. Rancho El Barbón, $50 \mathrm{~km}$ al NO de Ensenada, E. Guízar BC6 (ENCB). Mpio. Tecate. $10 \mathrm{~km}$ SW of Jacumé, R. Moran 24037 (MEXU). Mpio. Tijuana. $19 \mathrm{~km}$ E of Rosarito on road to San Borja, D.E. Breedlove 60971 (ENCB). S of Valle de Las Palmas, E of junction of highway 3 (Tecate - Ensenada) and road into Cerro Bola, near $2 \mathrm{~km} \mathrm{~S}$ of Valle de Las Palmas, R.F. Thorne et al. 62155 (MEXU). Tijuana, "Ag. Gral. De Agr.” sin fecha y sin número (MEXU). Mpio. Indefinido. Rocky slope along wash, 9 miles S of El Rosario, D.E. Breedlove, 43042 (MEXU). Laderas de la Sierra Juárez, San Miguel, E. Ezcurra et al. 31 marzo 1989 (MEXU). San José, C. Prof. J.M. Gallegos 27 noviembre 1922 (MEXU). 3 miles S of rancho Neji, I.L. Wiggins 11841 (MEXU). Baja California Sur: Mpio. Mulegé. 2-3 km NO de Santa Rosalillita, D.E. Breedlove 60841 (ENCB). 
Ephedra compacta Rose, Contr. U.S. Natl. Herb. 12(7): 261. 1909. Tipo: México. Puebla: near Tehuacán. Hills west of town, 2 sep 1906, J.N. Rose y J.S. Rose 11274 (holotipo: US! http://botany.si.edu/types/showImage. cfm?mypic=00012115.jpg).

Arbustos compactos, erectos o extendidos, 0.3-0.5 m de altura. Tallos jóvenes de color verde grisáceo, volviéndose grises, glaucos, con numerosos surcos longitudinales; madera de tallos maduros de color café grisáceo, ligeramente fisurada y fragmentada. Ramas rígidas, duras, casi cilíndricas, de hasta $2.5 \mathrm{~mm}$ de grosor, opuestas o verticiladas en los nudos, ángulo de divergencia de aproximadamente 37 grados; entrenudos $0.5-3 \mathrm{~cm}$ de largo. Yemas terminales de aproximadamente 1.5 mm de largo, con ápice obtuso, cónicas. Hojas opuestas, 1.5-3 mm de largo, con ápice obtuso que surge a partir de un engrosamiento medio-dorsal, connadas en 1/2-7/8 de su longitud total; vaina papirácea, color café rojizo en etapas tempranas, volviéndose gris (pajiza en seco) y abriéndose, subpersistente. Estróbilos masculinos solitarios o en pares, en los nudos de las ramas jóvenes, lanceolados a elipsoides, $5.5 \mathrm{~mm}$ de largo casi sésiles o con pedúnculo de hasta $1 \mathrm{~mm}$; brácteas 5-6 pares, opuestas, obovadas, connadas desde la base hasta en 1/2 de su longitud total, 2.2-5.8 $\mathrm{mm}$ de largo, 1.9-5.2 mm de ancho, márgenes basales rosados; microsporangióforo cerca de $1.9 \mathrm{~mm}$ de largo, con 3 microsporangios sésiles o estipitados. Estróbilos femeninos solitarios o en pares en los nudos de ramas jóvenes, ovoides, 4-8 mm de largo, casi sésiles o sobre pedúnculos hasta $3 \mathrm{~mm}$ de largo; brácteas 3-5 pares, ovadas, 4-5 mm de largo, 3-5 mm de ancho, connadas en 1/8-3/4 de su longitud, rojas y suculentas cuando maduras. Semillas en pares, de forma circular en corte transversal, de color pardo claro a oscuro, casi lisas, 3.5-5.5 mm de largo, 2-3 mm de ancho, ligeramente excediendo en tamaño a las brácteas; pico micropilar recto, ligeramente exerto, la punta truncada.

Crece en terrenos calizos, yesosos y rocosos, en matorral xerófilo, pastizal y bosque de Quercus y Pinus. Alt. 1600-2800 m. Se distribuye en México (Aguascalientes, Chihuahua, Coahuila, Durango, Hidalgo, Nuevo León, Oaxaca, Puebla, Querétaro, San Luis Potosí, Tamaulipas, Veracruz y Zacatecas). Fig. 4.

Nombres comunes: no conocidos.

Ejemplares examinados. Aguascalientes: Mpio. Asientos. Near $20 \mathrm{~km}$ E of Rincón de Romos, road to Asientos, between Cerro Altamira and Cerro de San Juan, R. McVaugh 23697 (MEXU). 6 km NE de Tepezalá en las faldas del Cerro San Juan, 


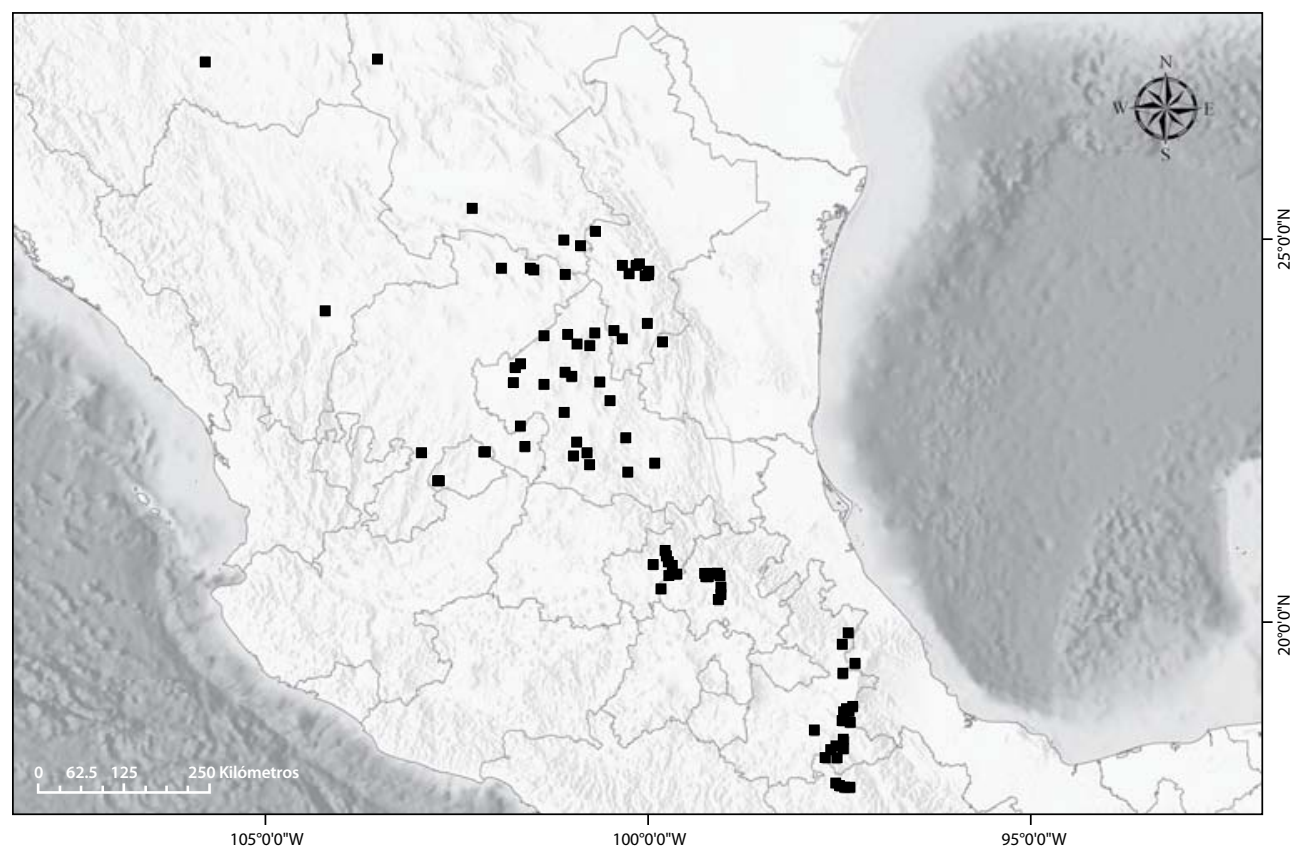

Fig. 4. Distribución de E. compacta en México.

V.M. Silva (25)085 (MEXU). $2 \mathrm{~km}$ al O de Asientos, cerca de la mina El Orito, J. Rzedowski 18254 (ENCB). Mpio. Calvillo. Cerca de Calvillo, F. Medellín 24776 (ENCB, MEXU, SLPM). Chihuahua: Mpio. Valle de Zaragoza. $22 \mathrm{~km} \mathrm{~S} \mathrm{of} \mathrm{Valle} \mathrm{de}$ Zaragoza on road to Hidalgo del Parral, M.C. Johnston et al. 11429A (MEXU). Coahuila: Mpio. Parras. Sierra de Parras; footrail from W part of town of Parras up to the highest part of the Sierra, M.C. Johnston et al. 11004 (MEXU). Mpio. Saltillo. Mountains S of Saltillo on road to Puerto de Flores, N. Boke y J. Massey 217 (MEXU). Llanura al O de Gómez Farías, A. Gómez, 669 (SLPM). Bajío de la U.A.A.”AN”, Buenavista, Saltillo, J.Á. Villarreal 2379 (MEXU). $50 \mathrm{~km}$ al S de Saltillo, carretera 54 a Concepción del Oro, entronque El Colorado, J.Á. Villarreal y J. Valdés R. 5688 (MEXU). Mpio. Sierra Mojada. Puerto Las Flores, A. Gómez 626 (MEXU). Durango: Mpio. Poanas. $3.5 \mathrm{~km}$ al N de La Cieneguilla, Y. Herrera 90 (ENCB, MEXU). Mpio. Indefinido. Al E de Vicente Guerrero, por el camino a Lobos, S. y M. González 1677 (MEXU, SLPM). Hidalgo: Mpio. Cardonal. $4 \mathrm{~km}$ al NO de Cardonal (El Mayorazgo), L. González 2512 (ENCB). $10 \mathrm{~km}$ al O de Cardonal, F. González-Medrano 8873 (ENCB, MEXU).Cuesta Blanca, 5 km al NE de Cardonal, E. Guízar 1468 (ENCB, MEXU). 19 km al E de Ixmiquilpan, R. Hernández 4616 (MEXU). Cerca de El Cubo, 
J. Rzedowski 49322 (MEXU). Camino de terracería Cardonal - La Mesa, F. Zavala 795 (MEXU). Mpio. Ixmiquilpan. Cañada de Arroyo Hondo, $25.9 \mathrm{~km}$ al NE de Ixmiquilpan, carretera a Tolantongo, R. Cruz et al. 4669 (MEXU). $8 \mathrm{~km}$ al E de Ixmilquilpan, L. González 3545 (ENCB), 3546 (ENCB). La Lagunita, C. Velasco y F. Ojeda 2 (MEXU). Mpio. San Salvador. $2 \mathrm{~km}$ al NO de Actopan, Cerro San Miguel de la Cal, L. González 3598 (ENCB), 3599 (ENCB). Mpio. Santiago de Anaya. $12 \mathrm{~km}$ al NO de Actopan, F. González-Medrano 3767 (MEXU). Nuevo León: Mpio. Dr. Arroyo. El Jarro, $16 \mathrm{~km}$ al O de Dr. Arroyo, F. González-Medrano et al. 4958 (MEXU). Mpio. Galeana. Highway 51, between Dr. Arroyo and Galeana, 34 km (by road) S of junction with mexican highway 58 at Puerto de Pastores and $58 \mathrm{~km} \mathrm{~N}$ of La Escondida, Breckon et al. 2283 (ENCB, MEXU). Sierra Madre Oriental, along the road to highway 57, about $15 \mathrm{~km} \mathrm{~S}$ of town of Galeana, Z. Debreczy et al. 39069B (MEXU). Along highway 58, about 0.5 miles $\mathrm{E}$ of El Carco, 5.9 miles $\mathrm{E}$ of junction road to Santo Domingo, about 6.3 miles E of Tokio, 10 miles E of highway 57 at San Roberto, M. Fishbein et al. 2955 (MEXU). $14 \mathrm{~km}$ by winding road W of Tokio on the San Roberto - Galeana highway, M.C. Johnston et al. 11052 (MEXU). Sierra Madre Oriental; foothills below Pablillo, a ranch 15 miles SE of Pueblo Galeana, C.H. Mueller 529 (MEXU). Arroyo Hondo, Hacienda San José de Raíces, C.H. Mueller 2309A (MEXU). Borde de carretera 57, tramo San Antonio de las Alazanas a Matehuala, $5 \mathrm{~km}$ al $\mathrm{N}$ de crucero a El Cuinje, al N de Navidad, R. Puente 2238 (SLPM). Camino de Pablillo a Cieneguillas $20 \mathrm{~km}$ al SO de Sierra Madre Oriental, sin colector 46 (MEXU). Mpio. General Zaragoza. Near $30 \mathrm{~km}$ ENE of Dr. Arroyo, ENE of San Antonio de Peña Nevada, W base of Cerro Peña Nevada, G. Nesom 4737 (MEXU). Oaxaca: Mpio. San Francisco Teopan, entre Ihuitlan, Concepción Buenavista, Tepelmeme y La Torrecilla, M. Cabrera 27 (MEXU). Mpio. San Miguel Tequixtepec. $2 \mathrm{~km}$ al S de Tepelmeme, distrito de Coixtlahuaca, J. Rzedowski 25758 (ENCB), 25759 (ENCB). Mpio. Santiago Chazumba. $5.5 \mathrm{~km}$ al N de Santiago Chazumba, F. González-Medrano et al. 11617 (MEXU). Mpio. Tepelmeme Villa de Morelos. Distrito Coixtlahuaca, Mixteca, S. Acosta 2447 (ENCB). $20 \mathrm{~km}$ al SE de Concepción Buenavista, carretera Tehuacán - Oaxaca, A. García-Mendoza et al. 7528 (MEXU). Mpio. Tlacotepec Plumas. 1 mile S of Tepelmeme de Morelos, by the unpaved road towards Tamazulapan, O. Dorado et al. 1654 (MEXU). Puebla: Mpio. Atexcal. $2 \mathrm{~km}$ al NO del poblado de San Juan Raya, A. Valiente et al. 168 (MEXU). Mpio. Atzitzintla. $9 \mathrm{~km}$ al NE de la caseta de cobro a Esperanza, $P$. Tenorio et al. 15861 (MEXU). Mpio. Caltepec. Al SE de Acatepec, P. Tenorio et al. 17308A (SLPM). Mpio. Cañada Morelos. $8 \mathrm{~km}$ al N-O de Azumbilla, carretera Tehuacán - Esperanza, A. Salinas et al. F-4004 (MEXU). Mpio. Chapulco. $10 \mathrm{~km}$ al NE de Azumbilla por la 
carretera rumbo a Cañada Morelos, F. Chiang et al. F-2639 (MEXU). Mpio. Esperanza. $4 \mathrm{~km}$ al SE de Esperanza, P. Dávila et al. 77 (ENCB). Mpio. Guadalupe Victoria. Laguna de Atexcac, San Luis Atexcac, R. Cruz 2711 (MEXU). Mpio. Palmar de Bravo. Cerro situado al $\mathrm{S}$ de la carretera Puebla - Orizaba, $8.6 \mathrm{~km}$ antes de llegar a la caseta La Esperanza, M. Ortiz-Olguín, 234 (ENCB). Cerro Boludo, ladera frente al pueblo de Guadalupe Piletas, A. Ramírez-Roa et al. 60 (MEXU). Mpio. Santa Ana Teloxtoc, E. Guízar y A. Castañeda 3969 (MEXU). Mpio. Tehuacán. 2 km al O de Tehuacán, F. Chiang et al. F-43 (MEXU). Meseta de San Lorenzo, subiendo por el camino que viene de El Riego, F. Chiang et al. F-2266 (MEXU). Mpio. Teziutlán. Barranca de Atexcaco, al N de Teziutlán, D. Gold 255 (MEXU). Mpio. Tlacotepec de Benito Juárez. $7 \mathrm{~km}$ al S de Tlacotepec, $2-3 \mathrm{~km}$ al N de Cacaloapan, A. Salinas et al. 7086 (MEXU). Mpio. Zapotitlán. Camino a Zapotitlán, SO de Tehuacán, F. Miranda 4434 (MEXU). Mpio. Indefinido. Km 150 carretera Puebla - Orizaba, G. Jiménez 9 febrero 1989 (MEXU). Km 207 Carretera México - Orizaba, A.R. López-Ferrari y A. Espejo 401 (MEXU). Querétaro: Mpio. Cadereyta de Montes. Entre Vizarrón e Higuerilla, Dr. Altamirano 1675 (MEXU). 18 km al NE de San Pablo Tolimán, sobre la brecha a Higuerillas, S. Zamudio 2255 (MEXU). 4 km al S del Vizarrón S. Zamudio 3659 (ENCB, MEXU). Aproximadamente $5 \mathrm{~km}$ al S de Vizarrón, S. Zamudio y V. Steinmann 12446 (MEXU). Mpio. Tolimán. San Antonio de la Cal, R. Hernández 12082 (MEXU). San Luis Potosí: Mpio. Catorce. Parte alta del Cerro Quemado, E. Pérez C. y S. Zamudio 3037 (MEXU). Aproximadamente $3 \mathrm{~km}$ al SE de Mineral de Catorce, J. Rzedowski 7285 (ENCB). Mpio. Cedral. Rancho San Miguel, $40 \mathrm{~km}$ al O de Charcas, A. Gómez 165 (SLPM). Mpio. Cerritos. Camino de Villa Juárez, Puerta del Río, A. Gómez 24169 (ENCB). Mpio. Cerro de San Pedro. Cerro San Pedro, al E del panteón, C. Méndez 75 (SLPM). Parte baja del Cerro de San Pedro, C. Méndez 25 abril 1997 (SLPM). 1 km al N de Cerro de San Pedro, J. Rzedowski 8221 (SLPM). Mpio. Charcas. Arroyo del Tepetclitle, cerro del Cerro, ejido de Milpitas, F. Medellín 28 octubre 1965 (SLPM). $14 \mathrm{~km}$ de Matehuala desde el entronque por la carretera a Cedral, A. Reyes 1639 (SLPM). Laguna Seca, J. Rzedowski 6507 (ENCB, SLPM). Mpio. Guadalcázar. Cerro El Coyote, por el camino que va hacia El Estribo, C. Méndez 69 (SLPM). Frente a la puerta principal del panteón, C. Méndez 74 (SLPM). Mpio. Moctezuma. Moctezuma, J. Villa 27179 (MEXU), J. Villa 39598 (ENCB). Mpio. Rioverde. Arroyo del Tepetclitle en el Cerro del Momo ejido de Milpitas, F. Medellín-Leal 24043 (MEXU). Rioverde. La Muralla, F. Takaki 24791 (MEXU). Santa Rosa, J. Villa 18 agosto 1964 (SLPM). Mpio. Salinas. Entre Tecomate y Salinas. Carretera San Luis Potosí - Salinas, A. Gómez 735 (SLPM). Mpio. San Luis Potosí. Km 127 carretera San Luis Potosí - Matehuala, a 100 m del camino, E. García 26 
marzo 1983 (SLPM). Mpio. Santo Domingo. 7 km al N de Herradura, A. Gómez 764 (ENCB). Estación Laguna Seca, B. Juárez agosto 1987 (SLPM). Por el camino Juan Sarabia hacia El Ciriaco, C. Méndez 66 (SLPM). Aproximadamente 3 km al NE de Peñasco, S. Salas, 404 (ENCB). Mpio. Sierra de Montegrande. Loma del Estañero, J.A. Reyes 983 (SLPM). Sierra de Montegrande, camino o brecha hacia Montegrande, a la altura de las faldas SE del cerro del Tunalillo, J.A. Reyes 1051 (SLPM). Mpio. Villa de Guadalupe. Santa Rosa, F. Medellín 1378 (ENCB). Moctezuma, J. Villa 30 mayo (SLPM). Mpio. Villa de La Paz. Aproximadamente al SO de Limones, F. Medellín 24783 (SLPM). Mpio. Zaragoza. San Francisco del Refugio - Palula, A. Gómez 659 (ENCB, SLPM). Mpio. Indefinido. El Salto, al O de la Sierra de la Chiscoa, C. Méndez 53 (SLPM), 54 (SLPM). Tamaulipas: Mpio. Miquihuana. $20 \mathrm{~km}$ al N de la Perdida, F. González-Medrano y P. Hiriart 13223 (MEXU). Veracruz: Mpio. Perote. Cerros, carretera 140, 2 km del límite con Puebla, L.I. Nevling y F. Chiang 1742 (MEXU). Cerros cerca de la carretera México - Veracruz, cerca del límite con el estado de Puebla, C.H. Ramos 147 (MEXU). Mpio. Indefinido. Cerro Yolotepec, al NE de San José Alchichica, F. González-Medrano 1608 (MEXU). Zacatecas: Mpio. Concepción del Oro. Mazapil. 1.5 km ESE of Salverna, on road to Concepción del Oro, F. Chiang et al. 7935 (MEXU). Foothills, Sierra del Astillero, $7 \mathrm{~km} \mathrm{~N}$ of Tanque El Alto, M.C. Johnston et al. 11577 (MEXU). Nearly 10.5 miles form Concepción del Oro on road to Mazapil, E. Lehto et al. L21766 (MEXU). Camino Concepción del Oro - Mazapil, M.F. Robert y J. Passini 5862 (ENCB). Mpio. Pinos. 1 km al O de Pinos, F. Gómez 715 (SLPM); S. Salas 585 (ENCB, SLPM).

Ephedra nevadensis S. Watson, Proc. Amer. Acad. Arts 14: 298. 1879. Tipo: Estados Unidos. Nevada: Smoky Valley, jul 1868, S. Watson 1108 pro parte (lectotipo: GH! código de barras 22597, designado por Cutler, 1939; isolectotipos: NY! US!). Ephedra antisyphilitica S. Watson var. pedunculata S. Watson, Bot. King Exped. 5: 330. 1871. Tipo: Estados Unidos. Nevada: sandy desert near Walker River, 7 jun 1859, H. Engelmann s.n. (lectotipo MO! designado por Cutler) http://www.tropicos. org/Image/10012359). Ephedra nevadensis S. Watson subvar. pluribracteata Stapf, Denkschr. Kaiserl. Akad. Wiss., Wien. Math.-Naturwiss. Kl. 56(2): 83. 1889. Tipo: Estados Unidos. California: Mohave River, southeast California, 1 jun 1876, E. Palmer 524 (sintipo: NY! http://sweetgum.nybg.org/vh/specimen.php?irn=47796).

Arbustos erectos, 0.25-1.25 m de altura. Tallos jóvenes de color verde pálido, glaucos, volviéndose amarillos, después grises, casi lisos o ligeramente ásperos, con pequeños surcos longitudinales; madera cinérea, fisurada. Ramas rígidas a flexuosas, 
sólidas, cilíndricas, de hasta 4 mm de grosor, opuestas o verticiladas en los nudos, con ángulo de divergencia de aproximadamente 45 grados; entrenudos de 1.5-6 cm de largo. Yemas terminales de 1-3 mm de largo con ápice obtuso, cónicas. Hojas opuestas, rara vez en verticilos de tres, 2-4 $\mathrm{mm}$ de largo, rara vez hasta $8 \mathrm{~mm}$, con ápice obtuso que surge a partir de un engrosamiento medio-dorsal, connadas en 1/2-2/3 de su longitud total, vaina completamente decidua. Estróbilos masculinos solitarios a varios en los nudos de las ramas jóvenes, elipsoides, 4-8 mm de largo, sésiles a cortamente pedunculados; brácteas 5-9 pares, opuestas, basales, connadas, obovadas, 3-4 mm de largo, 2-3 mm de ancho, membranáceas, de color amarillo a pardo claro, el verticilo inferior vacío; microsporangióforo 3-5 mm de largo, exerto 1/4-1/2 de su longitud, con 6-9 microsporangios sésiles o con un estípite muy corto. Estróbilos femeninos solitarios a varios en los nudos de las ramas jóvenes, subglobosos, 5-11 mm de largo; pedúnculo casi desnudo, 1-50 mm de largo; brácteas 3-5 pares opuestas, connadas en la base, 4-8 mm de largo, 3-6 mm de ancho, herbáceas, de color pardo claro a amarillo verdosas, ocasionalmente con un poco de rosa. Semillas en pares, rara vez solitarias, ligeramente tetragonales en corte transversal, pardas a casi negras, lisas, 6-9 mm de largo, 2-4 mm de ancho, de igual tamaño o excediendo a las brácteas; pico micropilar moderadamente exerto, ligeramente recurvado, escasamente contorto.

Cutler (1939) designó como lectotipo el ejemplar Watson 1108 (GH), en el cual se incluyen estróbilos femeninos de Ephedra viridis y estróbilos masculinos de Ephedra nevadensis.

Crece en terrenos calizos, en matorral xerófilo. Alt. 1215-1600 m. Se ha registrado de Estados Unidos de América (Arizona, California, Nevada, Oregon, Utah) y México (Baja California). Se ha mencionado su uso como remedio contra padecimientos renales. Fig. 5.

Nombres comunes: no conocidos.

Ejemplares examinados. Baja California: Mpio. Mexicali. Cerro Chichi de la India, R. Moran 30847 (ENCB). Mpio. Tecate. Shallow valley 8 km SE of La Rumorosa, R. Moran 30863 (MEXU).

Ephedra pedunculata Engelm. ex S. Watson, Proc. Amer. Acad. Arts 18: 157. 1883. Tipo: Estados Unidos. Texas: 90 miles northwest of San Antonio, oct 1880, E. Palmer 1291 (lectotipo: GH código de barras 22598, designado por Cutler, 1939; isolectotipos: NY! US!). 


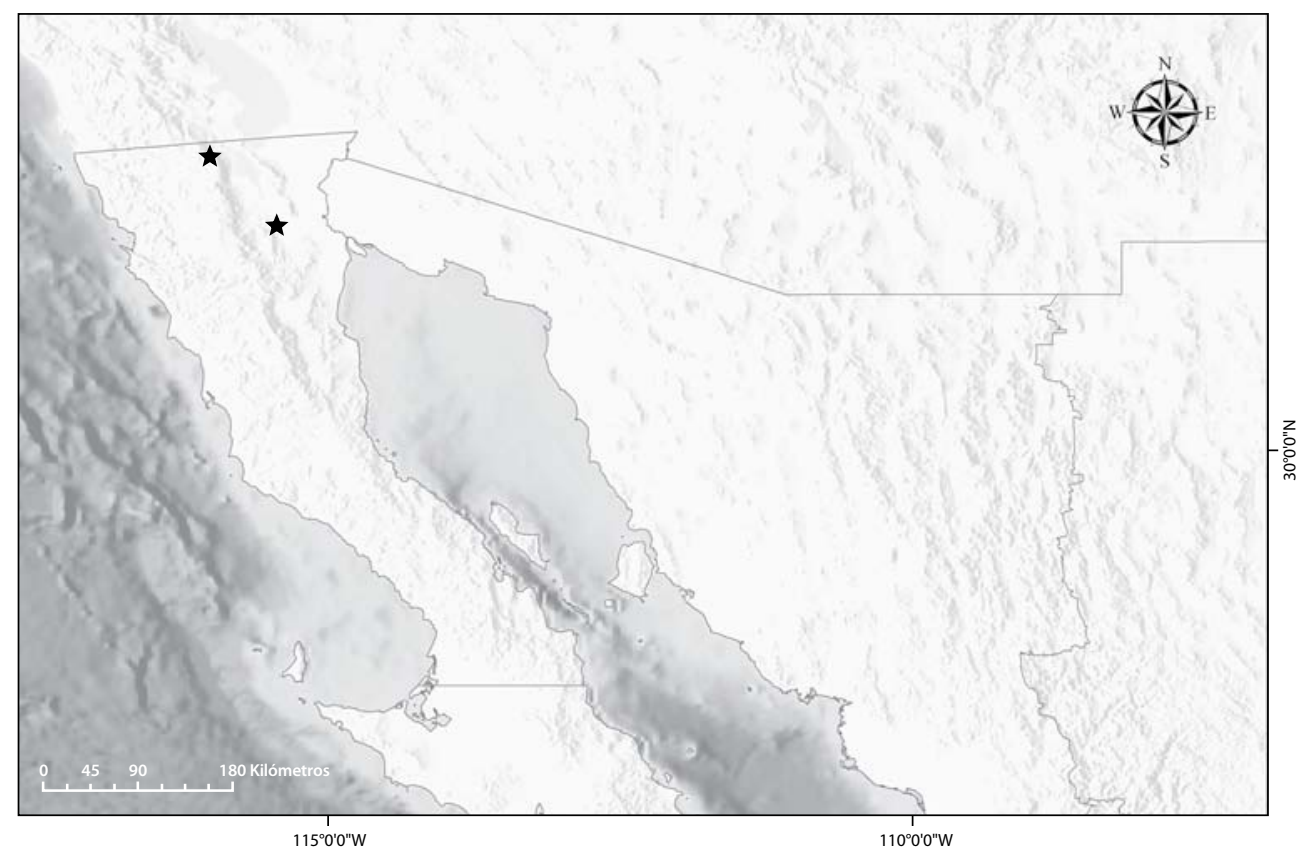

Fig. 5. Distribución de E. nevadensis en México.

Arbustos trepadores o postrados, usualmente con una altura de hasta 6-7 m. Tallos jóvenes color verde grisáceo, volviéndose de un color verde más intenso, después verde amarillento, glaucos, casi lisos o con varios surcos longitudinales profundos, la madera de tallos maduros cinérea, ligeramente fragmentada y fisurada. Ramas laxas, cilíndricas, de hasta $3 \mathrm{~mm}$ de grosor, alternas o rara vez verticiladas en los nudos, ángulo de divergencia de aproximadamente 52 grados, entrenudos 1-8 cm de largo. Yemas terminales 1-3 mm de largo, atenuadas. Hojas opuestas, 1-3 mm de largo, con punta obtusa que surge a partir de un engrosamiento herbáceo medio-dorsal, connadas desde 2/3 hasta 9/10 de su longitud total; vaina membranácea, persistente, que posteriormente se abre. Estróbilos masculinos solitarios o en pares en los nudos de las ramas jóvenes, lanceolados a elipsoides, 4-8 mm de largo; pedúnculos 0-12 mm de largo; brácteas 6-12 pares, obovadas, libres o connadas 1/8 de su longitud desde la base, 1.5-3.5 mm de largo, 1.5-3 mm de ancho, ligeramente engrosadas, márgenes membranáceos, de color amarillo a rojo, el par inferior vacío; microsporangióforo 3-5 mm de largo, exerto en $1 / 2$ de su longitud, con 4-6 microsporangios definitivamente estipitados. Estróbilos femeninos solitarios o en pares en los nudos de ramas jóvenes, rara vez varios en un 
nudo, elipsoides, 6-10 mm de largo; pedúnculos 1-20 mm de largo; brácteas 3-6 pares, ovadas, connadas en 1/8-7/8 de su longitud, los pares internos volviéndose carnosos, rojos, suculentos cuando maduros. Semillas en pares, trígonas en corte transversal, de color pardo claro a avellana, lisas, 4-9 mm de largo, 2-3.5 mm de ancho, conspicuamente exertas; pico micropilar ligeramente torcido, algo exerto, limbo contorto.

De acuerdo con lo observado en campo, E. pedunculata se puede encontrar trepando sobre árboles y arbustos a manera de bejuco, en el pastizal de Bouteloua de San Luis Potosí se encontró a la sombra, creciendo sobre los escasos individuos de Celtis sp. y Prosopis sp.

Crece en sustratos calizos y yesosos, en matorral xerófilo y pastizal. Alt. 3402125 m. Se ha registrado en Estados Unidos de América (SO de Texas) y México (Chihuahua, Coahuila, Durango, Nuevo León, San Luis Potosí, Tamaulipas, Zacatecas). En Zacatecas, según Palmer (en Conzatti, 1988) se usa como remedio contra pleuresía y neumonía. Fig. 6.

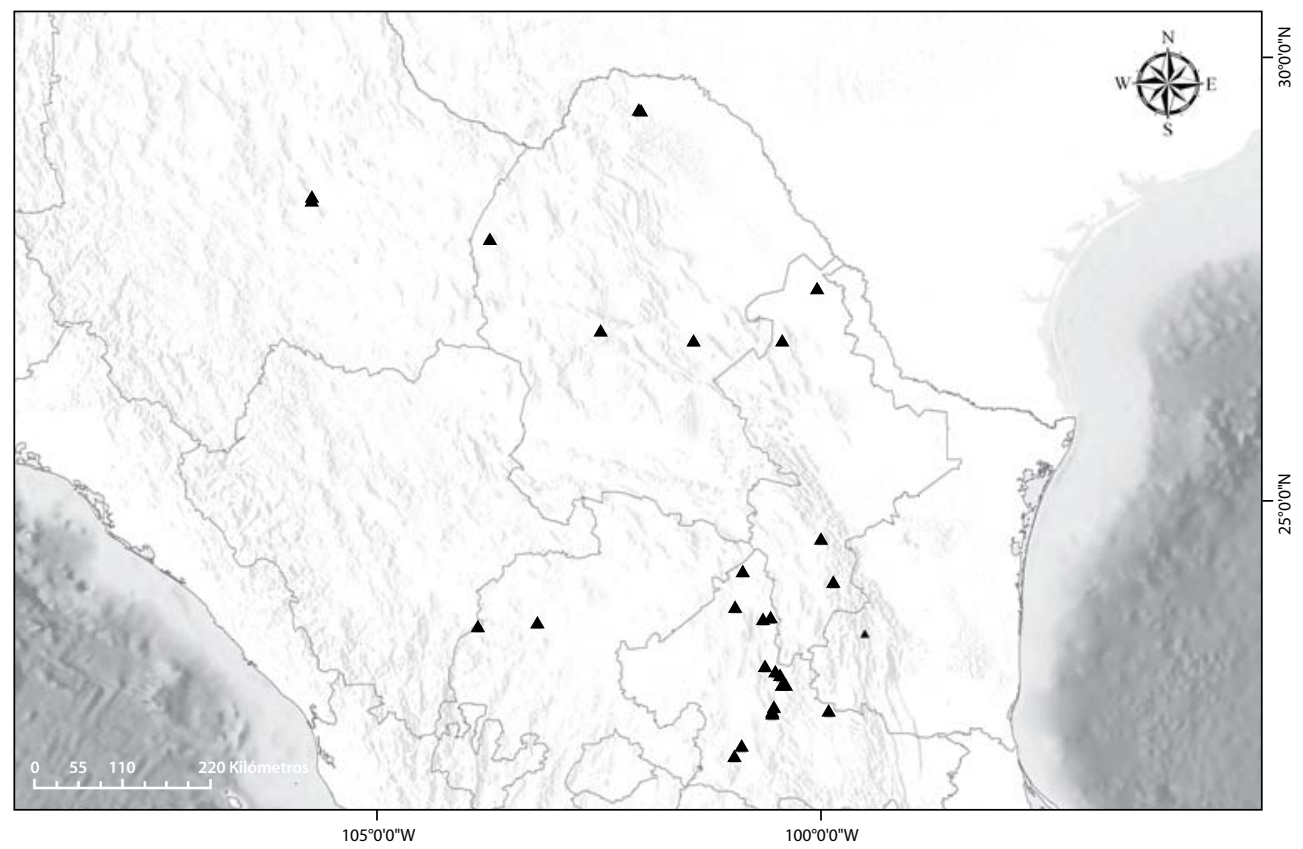

Fig. 6. Distribución de E. pedunculata en México. 
Nombres comunes: canatilla, comida de víbora, hintimoreal, itamoreal, pitamoreal, popotillo, retamo real, tepopote, sanguinaria.

Ejemplares examinados. Chihuahua: Mpio. Chihuahua. Rosales, 20 miles SE of Chihuahua on the road to Meoqui, M. C. Johnston 5271 (MEXU). Bachimba Cañón, C.G. Pringle 2 abril 1886 (MEXU). Coahuila: Mpio. Acuña. 25 km S by winding road from rancho Chupadero del Caballo toward Las Norias in Cañón del Colorado, F. Chiang et al. 9175 (MEXU). 42.5 km NE of road Las Norias and 29 km W of road Chupadero del Caballo, on the main road from C. Acuña to Huerfanita and La Linda, M.C. Johnston et al. 11739 (MEXU). Mpio. Castaños. Carretera Piedras Negras a Saltillo, $5 \mathrm{~km}$ al S del entronque carretera a Monterrey, al S de Castaños, $J$. Marroquín 2198 (ENCB, MEXU, SLPM). Mpio. Cuatro Ciénegas. Canyon de Fora, 7 $\mathrm{km} \mathrm{W}$ of Estación Socorro on the Cuatro Ciénegas - Esmeralda road, F. Chiang et al. 9149 (MEXU). Mpio. Progreso. 6 km al O de Progreso, L. Pimentel 22300 (ENCB). Mpio. Sierra Mojada. Vicinity of Santa Elena Mines, calcareous eastern foothills of the Sierra de Las Cruces, R.M. Stewart 2265 (MEXU), 2266 (MEXU). Durango: Mpio. Indefinido. Al SO de Súchil, cerca de los límites con estado de Zacatecas, S. González 1565 (SLPM). Nuevo León: Mpio. Aramberri. El Barreno, J.B. Hinton 17905 (ENCB). Mpio. Galeana. 1 km N of Pablillo, M.C. Johnston et al. 11044 (MEXU). Mpio. Lampazos. Rancho Reséndez, M.T. Edwards 385 (SLPM). San Luis Potosí: Mpio. Catorce. 4 km al S de Vanegas, F. Takaki 19 enero 1966 (SLPM). Mpio. Ciudad del Maíz. Charco Blanco, F. Medellín 652 (ENCB), 653 (MEXU, SLPM). Guadalcázar. Near km 549, 36 miles S of Matehuala, R. McVaugh 18203 (MEXU). Tepeyac, F. Takaki 18 julio 1966 (SLPM). Mpio. Matehuala. Ejido Cerrito Blanco, 10 $\mathrm{km}$ al E de Matehuala, S. Lemus 10 (ENCB, MEXU). Al N de Matehuala, L. Paray 2602 (ENCB). Mpio. San Luis Potosí. Alrededores de la ciudad, $4 \mathrm{~km}$ al NE, A. Rivera 102 (ENCB), 106 (MEXU). Mpio. Soledad de Graciano Sánchez. Vanegas. 2 $\mathrm{km}$ al NE de San Vicente, P. Castillo 23 febrero 1998 (SLPM). Villa de Guadalupe. Santa Rosa, J. Villa 18 agosto 1964 (SLPM). Km 144 de la carretera San Luis Potosí Matehuala, en el lado O, L. Villanueva y R.M. Fonseca 3 (FCME). Mpio. Indefinido. Km 50 carretera Huizache - Ciudad del Maíz, cerca del entronque de la Viga, J.L. Flores 21 octubre 1999 (SLPM). Entre Charco Blanco y Núñez, A. Gómez 23654 (SLPM). Los Núñez, $11 \mathrm{~km}$ al SO de la torre de la CFE, C. Méndez, 77 (SLPM). Km 15 carretera San Luis Potosí - Tampico, J. Rzedowski 3304 (ENCB, SLPM). Km 90 carretera San Luis Potosí - Matehuala, S. Salas 29 octubre 1996 (SLPM). Entre El Huizache y Matehuala, J. Valdés y F. Miranda A-61 (MEXU). A 1 km del Huizache, Villatana 28714 (SLPM). Km 144 de la carretera San Luis Potosí - Matehuala, S. 
Villegas y E. García 28018 (ENCB). Tamaulipas: Mpio. Jaumave. 2 km al SE de La Perdida, F. González-Medrano et al. 10320 (MEXU). Zacatecas: Mpio. Sain Alto. 58.6 km al N de Fresnillo, sobre carretera a Río Grande, después 7.3 km al O sobre camino a Nueva Rosita, en local de investigaciones de Mario González, al S del rancho El Carrizal, T. Wendt et al. 2188 (ENCB).

Ephedra torreyana S. Watson, Proc. Amer. Acad. Arts 14: 299. 1879. Tipo: Estados Unidos. Texas: sandy hills near Frontera, 26 abr 1851, C. Wright 1883 (lectotipo: GH! código de barras 22601, designado por Cutler, 1939; isolectotipos: MO! NY! US!).

Arbustos erectos, 0.25-1 m de altura. Tallos jóvenes de color verde azulado pálido, volviéndose grises, glaucos, casi lisos con numerosos surcos longitudinales; la madera de tallos maduros cinérea, fragmentada e irregularmente fisurada. Ramas rígidas, firmes, cilíndricas, de hasta $3.5 \mathrm{~mm}$ de grosor, solitarias o verticiladas en los nudos, con ángulo de divergencia de aproximadamente 48 grados; entrenudos 2-5 cm de largo. Yemas terminales de menos de $4 \mathrm{~mm}$ de largo, con ápice agudo, cónicas, no espinosas. Hojas deciduas, dispuestas en verticilos de tres, 2-5 mm de largo, con ápice obtuso u ocasionalmente agudo que surge a partir de un engrosamiento medio-dorsal, connadas en 1/3-2/3 de su longitud total, pero posteriormente abriéndose y recurvándose; vaina al principio membranácea, después fisurada, engrosada y grisácea, subpersistente. Estróbilos masculinos 1-4 en los nudos de las ramas jóvenes, ovoides, 6-8 mm de largo, sésiles; brácteas 6-9 en verticilos de 3, ovadas, ligeramente unguiculadas, 2-3.5 mm de longitud, 2-3 mm de ancho, membranáceas, de color crema a amarillo pálido, el verticilo inferior vacío; microsporangióforo de 2.5-4 mm de largo, exerto en 1/4-1/2 de su longitud total, con 5-8 microsporangios sésiles o cortamente estipitados. Estróbilos femeninos solitarios o varios en los verticilos en los nudos de las ramas jóvenes, ovoides, 9-13 mm de largo; sésiles; brácteas 5-6 en verticilos de 3, obovadas, unguiculadas, 6-9 mm de largo, 6-10 mm de ancho, hialinas excepto por la región central y basal, de color naranja amarillento a verde amarillento, márgenes dentados, ondulados. Semillas solitarias o en pares, ocasionalmente tres, trígonas o tetrágonas en corte transversal, de color pardo claro a verde amarillento, escabras, 7-10 $\mathrm{mm}$ de largo, $1.6-3 \mathrm{~mm}$ de ancho, igualando o excediendo ligeramente en tamaño a las brácteas; pico micropilar recto, conspicuamente exerto, limbo contorto, de $1 \mathrm{~mm}$ de largo.

En México se presenta Ephedra torreyana S. Watson var. powelliorum. 
Ephedra torreyana S. Watson var. powelliorum T. Wendt, Phytologia 74(2): 142. 1993. Tipo: México. Chihuahua: NE side of Sierra Cuchillo Parado, 4 abr 1976, M.C. Johnston, T. Wendt y F. Chiang 10579 (holotipo: TEX! 00370066 ; isotipos: MEXU! NY!).

Microsporangióforo con (2)3-5(7) microsporangios, todos sésiles o subsésiles, ocasionalmente los centrales sobre estípites de hasta $0.2 \mathrm{~mm}$ de largo. Semillas $1(-2)$ en cada estróbilo, lisas, en ocasiones rugosas en la base; pico micropilar exerto 0.3-1 mm por arriba del tegumento externo.

Crece en sustratos yesosos, en matorral xerófilo y dunas. Alt. 850-1400 m. Se ha registrado en Estados Unidos de América (Arizona, Colorado, Nevada y Texas) y México (Chihuahua). Fig. 7.

Nombres comunes: no conocidos.

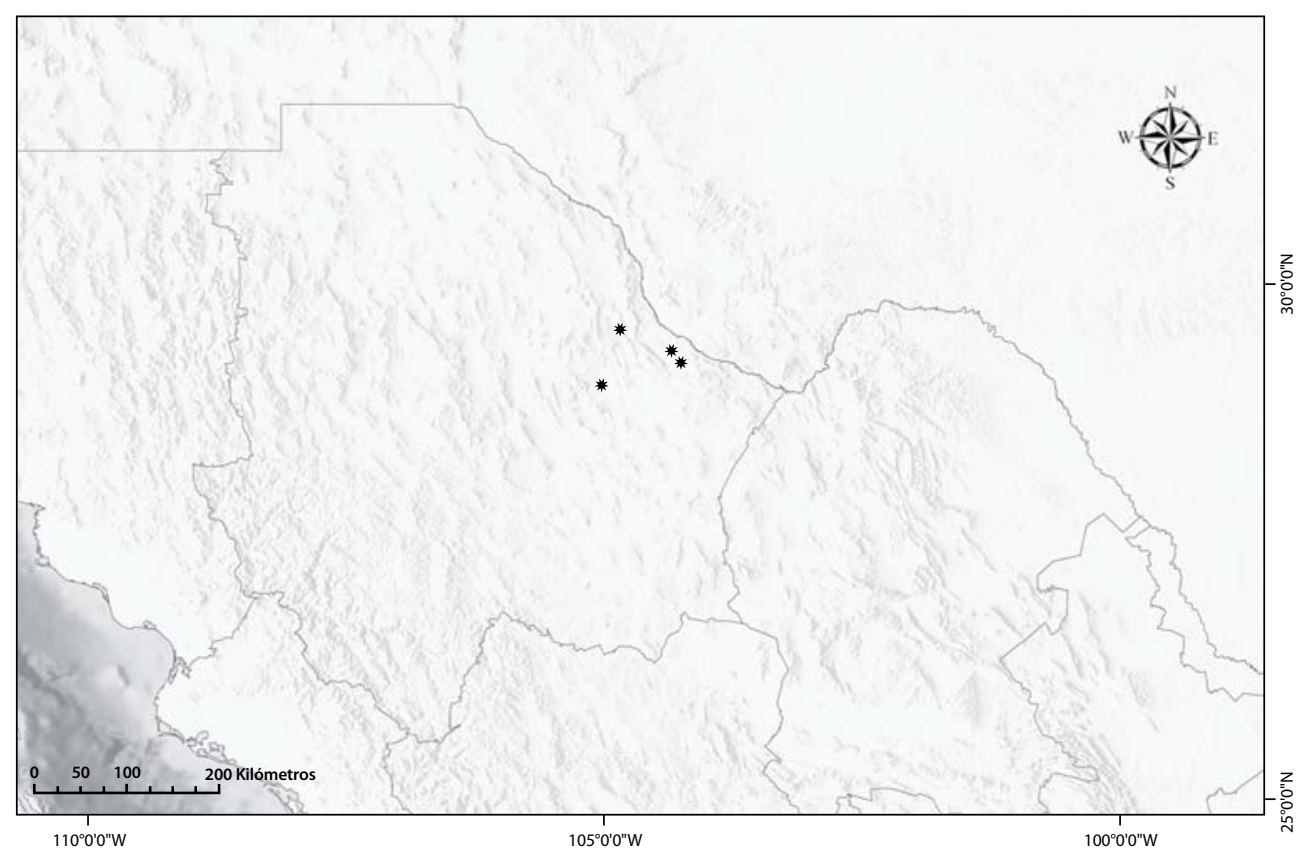

Fig. 7. Distribución de E. torreyana var. powelliorum en México. 
Ejemplares examinados. Chihuahua: Mpio. Ciudad Juárez. 6 km al NE de la Estación Samalayuca, R.D. Worthington 7259 (SLPM). Mpio. Coyame del Sotol. NE side of Sierra Cuchillo Parado, M.C. Johnston et al. 10578 (MEXU). NE side of Sierra Cuchillo Parado, M.C. Johnston et al. 10579 (MEXU). 4.7 miles S then E form Falomir railroad bridge along road to Chilicote, T. Wendt y E.J. Lott 770A (MEXU). Mpio. Ojinaga. 16 miles S of Ojinaga, along road to Camargo, H.S. Gentry y R. Engard 23198 (MEXU). $6 \mathrm{~km}$ E of Alamo Chapo Viejo on the road to La Consolación, M.C. Johnston et al. 10746 (MEXU), 10749 (MEXU).

Ephedra trifurca Torr. ex S. Watson, Bot. King Exped. 329. 1871. Tipo: Estados Unidos. Arizona: From the region between the Del Norte and the Gila, and the hills bordering the latter river to the desert west of the Colorado. Emory Exp. Tipo: no localizado.

Arbustos erectos, 0.5-2 m de altura. Tallos jóvenes de color verde pálido, volviéndose amarillos, después verde grisáceos, casi lisos, con numerosos surcos longitudinales; la madera de tallos maduros cinérea, fragmentada y fisurada longitudinalmente de manera irregular. Ramas rígidas, firmes, cilíndricas, de hasta 3.5 mm de grosor, solitarias o verticiladas en los nudos, con ángulo de divergencia de aproximadamente 30 grados; entrenudos 3-9 cm de largo. Yemas terminales $1 \mathrm{~cm}$ de largo, con ápice agudo, espinosas. Hojas dispuestas en verticilos de tres, 5-13 mm de largo, con ápice agudo que surge a partir de un engrosamiento medio-dorsal, connadas en 1/2-3/4 de su longitud total; vaina papirácea, después fibrosa, lacerada y grisácea, persistente. Estróbilos masculinos solitarios o numerosos en verticilos en los nudos de ramas jóvenes, obovoides, 6-9 mm de largo, cortamente pedunculados, pedúnculos escamosos; brácteas 8-12 en verticilos de 3, obovadas, ligeramente unguiculadas, 3-4 mm de longitud, 2-3 mm de ancho, membranáceas, de color pardo rojizo, los verticilos inferiores vacíos; microsporangióforo $4-5 \mathrm{~mm}$ de largo, exerto en $1 / 4$ de su longitud, con 4-5 microsporangios cortamente estipitados. Estróbilos femeninos solitarios o numerosos en verticilos en los nudos de las ramas jóvenes, obovoides, 10-14 mm de largo, sésiles o con un pedúnculo corto y escamoso; brácteas 6-9 en verticilos de 3, orbiculares, unguiculadas, 8-12 mm de largo, 9-12 mm de ancho, translúcidas excepto en el centro y la región basal que son de color pardo rojizo, márgenes enteros. Semillas solitarias, ocasionalmente en pares o grupos de tres, usualmente tetragonales en corte transversal, color pardo claro, lisas, 9-14 mm de largo, 1.5-3 mm de ancho, igualando en tamaño a las brácteas; pico micropilar conspicuamente exerto, limbo ligulado torcido, de $1 \mathrm{~mm}$ de largo. 
Crece en suelos yesosos y dunas, en matorral xerófilo y pastizal. Alt. 10-2100 m. Se distribuye en Estados Unidos de América (Texas y Nuevo México) y México (Baja California, Chihuahua, Coahuila, Durango, San Luis Potosí, Sonora y Zacatecas). Fig. 8.

Nombres comunes: no conocidos.

Ejemplares examinados. Baja California: Mpio. Ensenada. Mesa La Pitahaya, near 15 miles by road SE of El Rosario, G.L. Webster 26026 (MEXU). Mpio. Mexicali. Sierra de Las Pintas, H.S. Gentry 8737 (MEXU). Ejido Saldaña, 1 mile W on the road to Ejido Saldaña No. 2 from Mexico route 5 between Mexicali and San Felipe, J.P. Revman y J. Delgadillo 2231 (MEXU). Chihuahua: Mpio. Ahumada. 49.2 miles S of Ahumada on Mex. 45, W.E. Harmond y Dunn 5360 (ENCB). Mpio. Ascensión. Sand hills near Lake Guzmán, C.G. Pringle 7607 (MEXU). Mpio. Buenaventura. 28 miles W of junction route 45 and Road to Casas Grandes T. Stuessy 1111 (ENCB). Mpio. Camargo. $57 \mathrm{~km} \mathrm{~N}$ of Camargo - Jiménez highway on La Perla road; this is

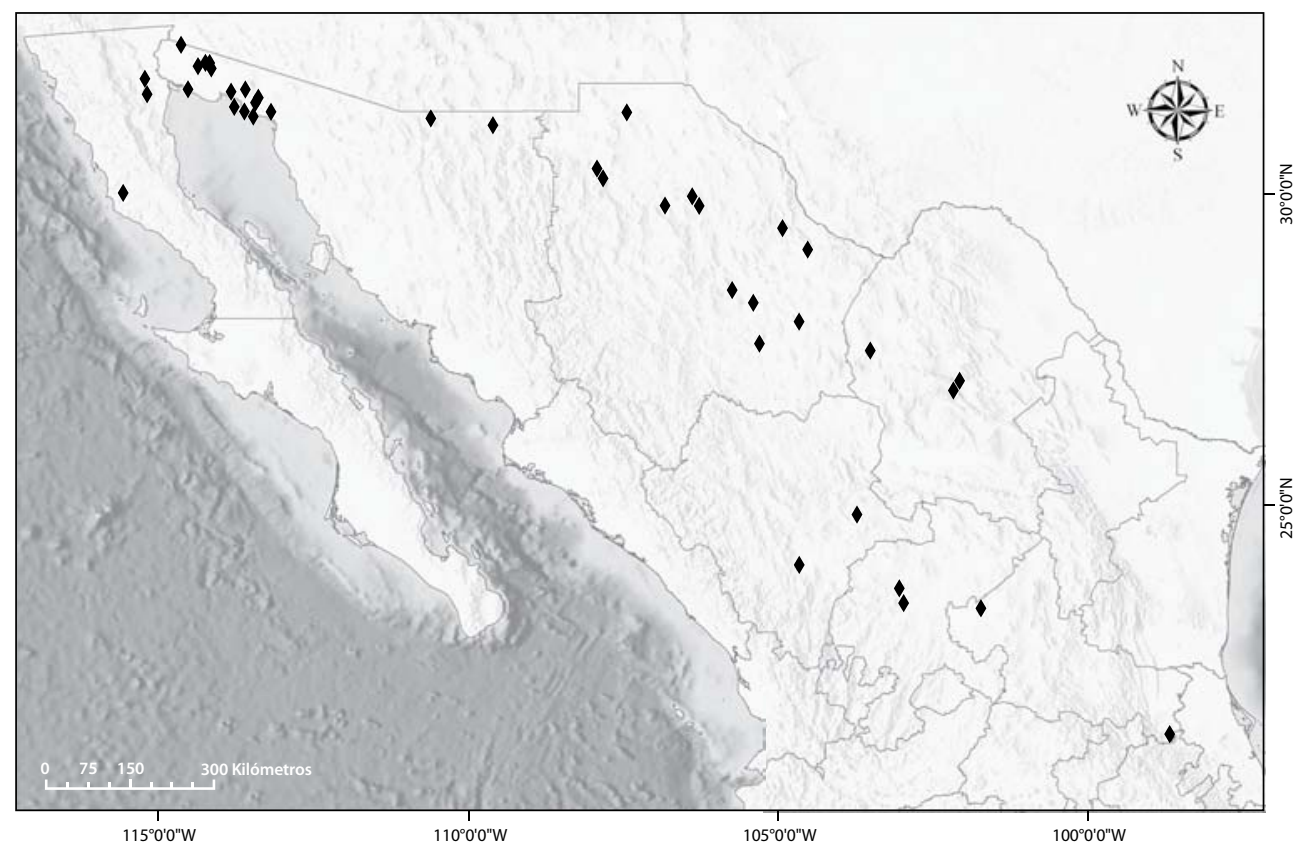

Fig. 8. Distribución de E. trifurca en México. 
$3 \mathrm{~km} \mathrm{~S}$ of junction of road departing eastward for San Fernando, M.C. Johnston et al. 10544 (MEXU). Mpio. Coyame del Sotol. 7 km E of Ejido Cuchillo Parado and on the far side of the Río Conchos, on the road to Coyame, M.C. Johnston et al. 10573 (MEXU). Mpio. Mesas. C.G. Pringle 1589 (MEXU). Mpio. Nuevo Casas Grandes. 10-15 miles SE of Nueva Casas Grandes, D.S. Correll y I.M. Johnston 21689 (MEXU). Nueva Casas Grandes - Colonia Juárez, I. Knobloch 375 (MEXU). 10 km antes de Casas Grandes, A. Vargas et al. 1 (ENCB, MEXU). Mpio. Ojinaga. 7.5 km S of Rancho San Luis on the Ojinaga - La Perla highway, M.C. Johnston et al. 10551 (MEXU). Mpio. Rosales. Al N del cerro La Aguja, G. Ibarra 1327 (MEXU). Mpio. San Francisco de Conchos. $14 \mathrm{~km} \mathrm{~W}$ of Camargo on highway to Lago Toronto, M.C. Johnston et al. 11407 (MEXU), 11407A (MEXU). Mpio. Indefinido. Colonia Cárdenas (Barranca Blanco) carretera Chihuahua - Ciudad Delicias, A. Becerra 12 abril 1955 (ENCB). Coahuila: Mpio. Cuatro Ciénegas. SO de Cuatro Ciénegas, J. Marroquín 2665 (ENCB). Poza "El Bonito", J. Marroquín 16 junio 1977 (ENCB). Bolsón de Mapimí, J.Z. Noguera BM-46 (MEXU). Laguna Grande, along road to dunes, at site of commercial removal of gypsum, D.J. Pinkava 5959 (ENCB). Durango: Mpio. Cuencamé. Cuencamé, F. Medellín 22 marzo 1973 (SLPM). Mpio. Durango. Durango, A. Ramírez 2898 (MEXU). San Luis Potosí: Mpio. Santo Domingo. Orilla N del Pueblo de Santo Domingo, A. Gómez 746 (ENCB, MEXU, SLPM). 2 km antes de Santo Domingo, por la carretera Charcas - Santo Domingo, C. Méndez 67 (SLPM). Sonora: Mpio. Agua Prieta. 15 miles by road S of Agua Prieta, R.S. Felger 3993, (MEXU). Mpio. Naco. $10 \mathrm{~km}$ al SE de Naco, P. Tenorio y C. Romero 13675 (MEXU). Mpio. Puerto Peñasco. Desierto de Altar, E. Ezcurra et al. 23 febrero 1989 (MEXU). Al SO de la Sierra del Pinacate, E. Ezcurra et al. sin fecha y sin número (MEXU). Strand at E side of mouth of Estero Morrua near 7.5 miles E of Puerto Peñasco, R.S. Felger y C. Baker 87-20 (MEXU). Near 1 mile S of Moon Crater (Crateo Chichi), SW part of Pinacate Region, R.S. Felger 19009 (ENCB).Upper end of Choya Bay, H. Hanson 7 febrero 1970 (ENCB). N of Cholla Bay, an American settlement, near 5 miles N of Town Puerto Peñasco, A.F. Johnson 4050 (MEXU). Building foredunes above beach at Estero Salado, A.F. Johnson 4067 (MEXU). Pinacate region, dunes 1 mile S of Luna Crater, G.L. Webster 24259 (MEXU). Mpio. San Luis Río Colorado. Near 2 miles SE of SE tip of Sierra del Rosario, Gran Desierto, H.T. Coss et al. 20364 (ENCB). Near 5 miles NE of Sierra del Rosario, Gran Desierto, H.T. Coss et al. 20437 (ENCB). Dunas de la costa del Golfo de Santa Clara, E. Ezcurra et al. 24 marzo 1989 (MEXU). 8 km W of main mountain mass of Sierra del Rosario, R.S. Felger et al. 75-36 (MEXU). Near $3 \mathrm{~km} \mathrm{~W}$ of main mountain mass of Sierra del Rosario, R.S. Felger et al. 75-43 (MEXU). Beach dunes $0.5 \mathrm{~km}$ E of El Golfo de 
Santa Clara, R.S. Felger 75-82 (MEXU). Near 2 miles N of Sierra del Rosario, Gran Desierto, R.S. Felger 20773 (ENCB). E of San Luis Río Colorado, F. Miranda, 8899 (MEXU). 15.4 km E of San Luis Río Colorado on Mex. 2, A.L. Rein 97-525 (MEXU). Mpio. Santa Cruz. Along road to Cajón Bonito, C.T. Mason 3181 (ENCB, MEXU). Zacatecas: Mpio. Fresnillo. 4 km al S de Rancho Grande, H. Puig 6603a (ENCB). Río Grande. 58.6 km al N de Fresnillo sobre carretera a Río Grande, después 7.3 km al O sobre camino a Nueva Rosita, en local de investigaciones de Mario González al S de rancho El Carrizal, T. Wendt y E. Lott 2195 (ENCB, MEXU).

\section{Distribución de las especies de Ephedra en México}

De aproximadamente 60 especies de Ephedra que existen en el mundo (Ickert-Bond y Wojciechowski, 2004), ocho se presentan en México, lo que constituye $12 \%$ del total; de éstas, siete se comparten con Estados Unidos de América y sólo Ephedra compacta se considera endémica de México. El área de distribución de estas especies coincide esencialmente con la de la región xerofítica mexicana.

Méndez (1998) registra a E. fasciculata A. Nelson, E. peninsularis I.M. Johnston y E. viridis Coville para San Luis Potosí; sin embargo E. peninsularis es un sinónimo de E. aspera. Respecto a Ephedra fasciculata, que fue reportada para algunas zonas de Sonora (Wiggins, 1980), no se consideró en este estudio ya que los tres ejemplares (R. Moran 19709, F. Giovanini 6 febrero 1976 y A.L. Haines 17 febrero 1939) presentan características afines a $E$. fasciculata pero difieren de ésta en cuanto al tamaño de la semilla. Por otra parte, debido a que tienen un mayor número de características de E. aspera, tales como color y textura de las ramas, filotaxia, tamaño de hojas y de semillas, se decidió considerarlas como parte de esta última especie. Tampoco se encontraron ejemplares de México con las características de E. viridis, planta con las ramas de color amarillo intenso y la base de las hojas persistentes. Por estas razones no se incluyó en este trabajo.

El género tiene una importante presencia en el territorio nacional pues, de acuerdo con los materiales revisados, se encuentra en 16 estados de la República Mexicana: Aguascalientes, Baja California, Baja California Sur, Chihuahua, Coahuila, Durango, Hidalgo, Nuevo León, Oaxaca, Puebla, Querétaro, San Luis Potosí, Sonora, Tamaulipas, Veracruz y Zacatecas. Fig. 9.

Ephedra compacta se registra de 13 de los 16 estados donde se distribuye el género. E. aspera de 11 y E. antisyphilitica de 10. Las especies de presencia más restringida en México son $E$. torreyana que sólo se ha colectado en Chihuahua, $E$. nevadensis que se ha encontrado únicamente en Baja California y E. californica sólo en Baja California y Baja California Sur. 
El análisis de los datos que acompañan a los ejemplares consultados sugiere que las especies mexicanas de Ephedra se encuentran preferentemente en suelos calizos o yesosos. Se pueden encontrar en un intervalo altitudinal que va desde el nivel del mar (E. californica) hasta altitudes que alcanzan los $2800 \mathrm{~m}$.

De acuerdo con las observaciones en el campo y con los datos de colecta de los ejemplares consultados, las ocho especies de Ephedra crecen en las zonas áridas y semiáridas, preferentemente en tipos de vegetación como el pastizal y matorrales xerófilos. Según Méndez (1998), en lo que respecta a San Luis Potosí, E. antisyphilitica, E. aspera, E. compacta y E. pedunculata, se establecen principalmente en matorral micrófilo, aunque también pueden hacerlo en el rosetófilo y en el crasicaule. De manera general, se puede decir que la información sobre el tipo de vegetación de los ejemplares de herbario es pobre, por lo que se hace hincapié para que en futuras colectas se registre este dato.

Desde el punto de vista fitogeográfico, Ephedra se ha ubicado tanto en el reino Holártico como en el Neotropical, en las cuatro regiones que Rzedowski (1978) define para México: Pacífica Norteamericana, Mesoamericana de Montaña, Xerofítica Mexicana y Caribea, así como en la mayoría de las 17 provincias florísticas que el mismo autor propone para el territorio mexicano. Las provincias Costa Pacífica, Islas Revillagigedo, Soconusco y Península de Yucatán no cuentan con registros del género.

Para la determinación taxonómica de ejemplares de este grupo es indispensable contar con materiales fértiles, pues los caracteres vegetativos con mucha frecuencia no son suficientes para distinguir entre especies. Esto es particularmente cierto para las plantas de hojas opuestas, como E. aspera y E. antisyphilitica. La identificación de ambas en el campo resulta relativamente fácil por el color de los tallos que en E. antisyphilitica es verde muy pálido, casi grisáceo, mientras que los de E. aspera son de color verde olivo intenso. En ejemplares de herbario la determinación no resulta tan sencilla y se debe prestar atención al color de los nudos, que son oscuros y abultados en E. aspera y pálidos en E. antisyphilitica donde, además, las hojas son deciduas y se rasgan en la madurez, mientras que en E. aspera las hojas son perennes.

Hasta el momento no se conoce con precisión la fenología del género, al menos en México. De acuerdo con los datos recabados en los ejemplares examinados, la presencia de estructuras fértiles se observa en distintos meses a lo largo del año. No obstante, al realizar visitas al campo en esos periodos, no siempre se encontraron ejemplares con estructuras reproductoras. I.M. Johnston (1943) menciona que durante las visitas que realizó entre los meses de julio y octubre, no encontró 
Villanueva-Almanza y Fonseca: Revisión taxonómica y distribución de Ephedra en México

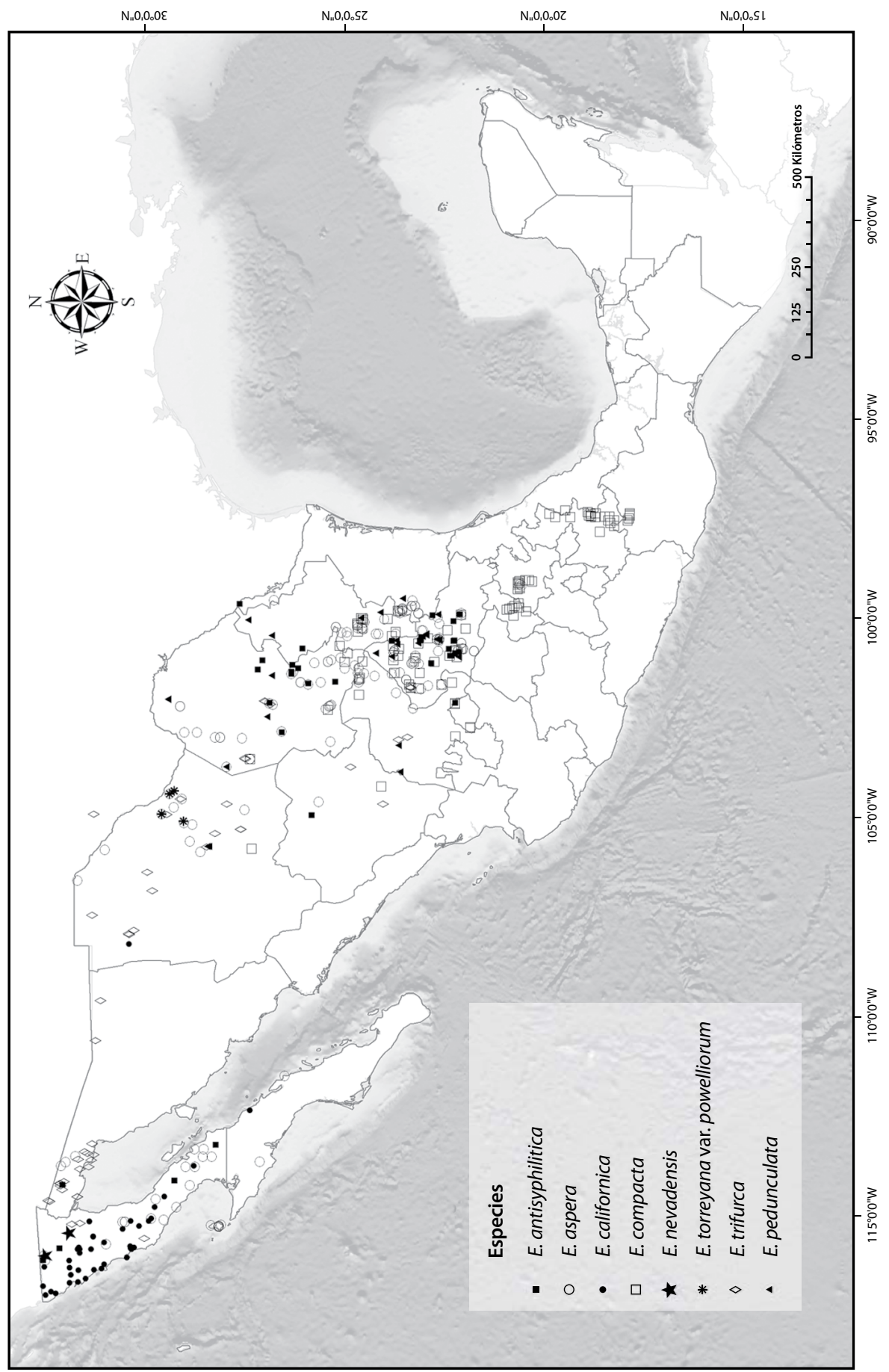

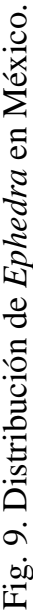


ejemplares fértiles de E. torreyana. Esta observación se puede aplicar también para las especies de E. antisyphilitica, E. aspera, E. compacta y E. pedunculata. Medina y Dávila (1997) reportan que E. compacta presenta estróbilos entre junio y noviembre, pero esto no pudo ser verificado en las visitas realizadas en el estado de Puebla.

Ephedra se presenta en regiones áridas y semiáridas y entre las características relacionadas con este tipo de ambientes se puede mencionar el hecho de que los tallos están recubiertos por una capa cerosa que les confiere un color grisáceo y muy probablemente refleja los rayos del sol, así como la ubicación de los estomas en los surcos de los tallos. Además poseen raíces profundas que permiten mayor captación de agua.

Ephedra antisyphilitica, E. compacta y E. pedunculata desarrollan brácteas carnosas. Hollander et al. (2009) sugieren que tal carácter se relaciona con la presencia de estas especies en zonas de mayor humedad que aquellas de brácteas secas.

Las visitas al campo permitieron observar que Ephedra compacta, E. aspera y E. antisyphilitica son frecuentemente ramoneadas por el ganado, se sabe también que Ephedra californica y E. nevadensis son utilizadas en Baja California como remedio para padecimientos renales; sin embargo, se requiere más información sobre los metabolitos secundarios de estas especies para hacer un buen aprovechamiento de este recurso.

Actualmente ninguna especie de Ephedra está mencionada en la Norma Oficial Mexicana NOM-050-ECOL-2001, ni por la Convención Internacional de Especies Amenazadas de Fauna y Flora Silvestre (CITES) como especie en riesgo.

\section{AGRADECIMIENTOS}

A la Dra. Rosa Irma Trejo del Instituto de Geografía por su apoyo para elaborar los mapas, a Ernesto Velázquez de la Facultad de Ciencias y a José García del Instituto de Investigaciones de Zonas Desérticas de la Universidad Autónoma de San Luis Potosí por su colaboración en el trabajo de campo, a Rosalinda Medina por la lectura crítica del manuscrito, a Pedro Gabriel Díaz Maeda por la elaboraciòn de los mapas, al personal de los herbarios ENCB, MEXU, SLPM y UAMIZ por permitir la consulta de las colecciones y a las señoras Rosa Silbata y Susana Rito del Instituto de Geografía por permitir la consulta de las cartas topográficas. 


\section{LITERATURA CITADA}

Caveney, S., D. A. Charlet, H. Freitag, M. Maier-Stolte y A. N. Starratt. 2001. New observations on the secondary chemistry of World Ephedra (Ephedraceae). Am. J. Bot. 88(7): 1199-1208.

Conzatti, C. 1988. Flora taxonómica mexicana. Volumen I. Consejo Nacional de Ciencia y Tecnología. México, D.F. 1064 pp.

Cutler, H. C. 1939. Monograph of the North American species of the genus Ephedra. Ann. Missouri Bot. Gard. 26: 373-426.

Hollander, J. L., S. B. Vander Wall y J. G. Baguley. 2009. Evolution of seed dispersal in North American Ephedra. Evol. Ecol. 23(4): 491-503.

Ickert-Bond, S. y M. F. Wojciechowski. 2004. Phylogenetic relationships in Ephedra (Gnetales): evidence from nuclear and choloplast DNA sequence data. Syst. Bot. 29(4): 834-849.

Johnston, I. M. 1943. Plants of Coahuila, Eastern Chihuahua and adjoining Zacatecas and Durango. I. J. Arnold Arbor. 24: 306-339.

Martens, P. 1971. Les gnétophytes. Handbuch der Pflanzenanatomie, Band 12, Teil 2. Gebrüder Borntraeger. Berlín-Stuttgart. 295 pp.

Medina, R. y P. Dávila. 1997. Gymnospermae. Flora del Valle de Tehuacán-Cuicatlán. Fasc.12. Instituto de Biología, Universidad Nacional Autónoma de México. México, D.F. pp. 14-17.

Méndez, C. 1998. Distribución geográfica y ecológica del género Ephedra L. en el Altiplano Potosino. Tesis de maestría en ciencias. Facultad de Agronomía, Universidad Autónoma de San Luis Potosí. San Luis Potosí, México. 90 pp.

Meyer, C. A. 1846. Versuch einer Monographie der Gattung Ephedra. Mem. Acad. Imp. Soc. St. Petersburg 6 Ser. 7: 225-298.

Pant, D. D. y B. K. Verma. 1974. Taxonomy of the genus Ephedra. Significance of stem and leaf epidermis and cuticle. Bot. J. Linn. Soc. 69: 287-308.

Rydin, C., K. Raunsgaard, P. R. Crane y E. M. Friis. 2006. Former diversity of Ephedra (Gnetales): evidence from Early Cretaceous seeds from Portugal and North America. Ann. Bot. 98(1): 123-140.

Rzedowski, J. 1978. La vegetación de México. Limusa. México, D.F. 432 pp.

Standley, P. C. 1923. Ephedra. In: Trees and shrubs of Mexico. Contr. U.S. Nat. Herb. 23(1): 63-64.

Stapf, O. 1889. Die Arten der Gattung Ephedra. Denkschr. Kaiserl. Akad. Wiss., Math.Naturwiss. Kl. 56(2): 1-112.

Stevenson, D. W. 1993. Ephedraceae. Flora of North America North of Mexico 2: 429-434.

Valdés, J. y H. Flores. 1987. Las gimnospermas en la flora halófila y gipsófila de México. An. Inst. Biol. Ser. Bot. 57: 45-58.

Wendt, T. 1993. A new variety of Ephedra torreyana (Ephedraceae) from west Texas and Chihuahua, with notes on hybridization in the E. torreyana complex. Phytologia 74(2): 141-150. 
Acta Botanica Mexicana 96: 79-116 (2011)

Wiggins, I. L. 1980. Ephedra. In: Flora of Baja California. Stanford University Press. California. 997-1025 pp.

Yang, Y. B., Y. Gneg, D. L. Dilcher, Z. D. Chen y T. A. Lott. 2005. Morphology and affinities of an Early Cretaceous Ephedra (Ephedraceae) from China. Am. J. Bot. 92: 231241.

Recibido en agosto de 2010.

Aceptado en junio de 2011. 